AperTO - Archivio Istituzionale Open Access dell'Università di Torino

\title{
Vibrational spectroscopy of hydrogens in diamond: A quantum mechanical treatment
}

\section{This is the author's manuscript}

Original Citation:

Availability:

This version is available http://hdl.handle.net/2318/1692578

since 2019-02-15T12:00:00Z

Published version:

DOI:10.1039/c8cp00596f

Terms of use:

Open Access

Anyone can freely access the full text of works made available as "Open Access". Works made available under a Creative Commons license can be used according to the terms and conditions of said license. Use of all other works requires consent of the right holder (author or publisher) if not exempted from copyright protection by the applicable law. 
Can hydrogen atoms saturate the four dangling bonds of the carbon atoms around the vacancy in diamond? A Quantum Mechanical investigation.

\author{
Francesco Silvio Gentile, ${ }^{1}$ Simone Salustro, ${ }^{1}$ Jacques Kontak \\ Desmarais, ${ }^{1}$ Anna Maria Ferrari, ${ }^{1}$ Silvia Casassa, ${ }^{1}$ and Roberto Dovesi ${ }^{1}$ \\ ${ }^{1}$ Dipartimento di Chimica, Università di Torino and \\ NIS (Nanostructured Interfaces and Surfaces) Centre, \\ Via P. Giuria 5, 10125 Torino, Italy
}




\begin{abstract}
\end{abstract}
aggiungere il bending e altre 2 limitations

$\mathrm{VH}_{n}$ ( $n=1$ to 4 ) defects in diamond (hydrogen atoms saturating the dangling bonds of the carbon atoms surrounding a vacancy) are investigated at the quantum mechanical level by using the periodic supercell approach, an all electron gaussian type basis set, "hybrid" functionals, and the CRYstal code. Most of the results have been collected for $\mathrm{S}_{64}$ (a supercell containing 64 atoms). However, for the cases with the highest $\mathrm{H}$ concentration, $\mathrm{VH}_{4}$, also the $\mathrm{S}_{216}, \mathrm{~S}_{512}$ and $\mathrm{S}_{1000}$ supercells have been considered, to explore the effect of the defect concentration on the IR and Raman spectra. Various spin states are considered $\left(\mathrm{S}_{z}=1 / 2\right.$ for $\mathrm{VH}_{1} ; \mathrm{S}_{z}=1$ and 0 for $\mathrm{VH}_{2}$ and $\mathrm{V}$, the pure vacancy, here included for comparison; $\mathrm{S}_{z}=1 / 2$ and $3 / 2$ for $\mathrm{VH}_{3}$ and $\mathrm{S}_{z}=0$ for $\mathrm{VH}_{4}$ ): their relative stability, band structure, charge and spin density distributions are analyzed. All the investigated systems present specific IR and Raman spectra, with peaks that can be used for the characterization of the defects. In particular the $\mathrm{CH}$ stretching peaks can be considered fingerprints of the system: they range (when anharmonicity is taken into account) from 2500 to $4400 \mathrm{~cm}^{-1}$ as a function of the number of $\mathrm{H}$ atoms in the vacancy. Also the dependence on the spin state is remarkable: for $\mathrm{VH}_{1}$, its harmonic frequency increases from about $2800 \mathrm{~cm}^{-1}$ for the quadruplet state to about $3200 \mathrm{~cm}^{-1}$ for the doublet. The accurate determination of these vibrational frequencies requires two main ingredients, that have been used in the present study: i) "hybrid" functionals, that are known to produce accurate vibrational frequencies; ii) a specific treatment for the determination of the stretching $\mathrm{CH}$ anharmonicity. The latter too is extremely sensitive to the $\mathrm{H}$ load and spin state of the system, ranging from $-340 \mathrm{~cm}^{-1}$ (the lowering of the wavenumber with respect to the harmonic value in $\mathrm{VH}_{1}$ ) to $+84 \mathrm{~cm}^{-1}$ for $\mathrm{VH}_{4}$, where the anharmonic wavenumber increases with respect to the harmonic value due to the strong repulsion with the other $\mathrm{H}$ atoms. The graphical animation of the modes (see http://www.pmmp.jussieu. $\mathrm{fr} / \mathrm{yves} /$ defectsindiamond/) permits to investigate the nature of the full set of modes.

Keywords: Diamond, 1 to $4 \mathrm{H}$ in the vacancy, electronic structure, formation energy, IR spectrum, anharmonicity, animation of the vibrational modes, isotopic substitution 


\section{INTRODUCTION}

aggiungere il bending

Hydrogen is one of the most important impurities in diamond. It is included during the growth of natural diamond as well as in chemical vapour deposition (CVD) processes. It is certainly present at the surfaces; a non minor fraction, however, is also thought to be incorporated in the bulk. $\mathrm{H}$ atoms are supposed to be present in several defects ${ }^{1,2}$, involving vacancies and/or nitrogen atoms isolated or aggregated. It is mostly identified through its infrared (IR) active modes (stretching and bending), although probably not all hydrogen atoms present in diamond are IR active. A general review concerning hydrogen in diamond has been published in 2002 by Briddon and coworkers ${ }^{3}$, that explores a set of hydrogen containing defects from the simulation point of view. Five years later a second review paper appeared $^{4}$, that gives an overview, from the experimental side, of the peaks that "have been at least tentatively attributed to H-related defects". ${ }^{4}$ As a matter of fact, the one to one correspondence between the many peaks that can reasonably be attributed to $\mathrm{H}$ (some of the wavenumbers larger than say $1400 \mathrm{~cm}^{-1}$ ) and a specific local defect is still lacking. For example, only very recently (in 2014) Briddon and coworkers have been able to attribute to the $\mathrm{VN}_{3} \mathrm{H}$ defect ${ }^{5}$ (a vacancy surrounded by three $\mathrm{N}$ and a $\mathrm{C}$ atoms saturated by $\mathrm{H}$ ) the peaks at 1405 and $3107 \mathrm{~cm}^{-1}$, that are observed in almost all types of Ia natural diamonds ${ }^{1,4,6-8}$. In particular, and with reference to the present investigation, we have been unable to find indications concerning the number of $\mathrm{H}$ atoms that can enter in the vacancy: is there enough space for two, or three, or four $\mathrm{H}$ atoms saturating the $\mathrm{C}$ dangling bonds? Do the progressive saturation remain exothermic up to 3 or $4 \mathrm{H}$ atoms, or is the steric hindrance preventing the high loading? How different is the IR spectrum of the various $\mathrm{VH}_{n}$ compounds and spin states? Is the Raman spectrum adding complementary information?

From the experimental side, it is obviously very difficult to know how many different defects containing $\mathrm{H}$ are present, and at which concentration, and how combined with other defects. From the simulation point of view, where it is possible to define a priori the kind of defects present in the bulk, other limitations prevent however from a simple identification of the experimental peaks. The four most important limitations are related to a) the use of functionals (LDA, GGA) that are known to perform poorly with open shell systems, and in 
describing $\mathrm{H}$ atoms for which the self interaction error is particularly severe ${ }^{9}$; b) inaccuracies in the models for treating the vibrational modes, that in the particular case of the C-H stretching is very anharmonic; c) the non availability of the IR intensities, that permit to complement the wavenumber calculation for producing the full spectrum and d) difficulties in modeling large unit cells or clusters, under the hypothesis that the experimental data refer to "isolated" defects, where the mutual interaction is supposed to be negligible. Some of these limitations deeply affect the previous quantum mechanical simulations of $\mathrm{H}$ containing defects.

Here we extend our preliminary investigation devoted to $\mathrm{VH}_{1}{ }^{10}$, by considering the full set of $\mathrm{VH}_{n}$ ( $n=1$ to 4$)$ defects, with significant improvements with respect to previous treatments (the four limitations mentioned above are totally or in part eliminated). One, two, three and four dangling bonds of $\mathrm{V}$ (the diamond vacancy) are saturated, by using a supercell approach, hybrid functionals (most of the calculations will be performed with B3LYP), a Gaussian type basis set and the supercell scheme. Cells containing 64 atoms, $\mathrm{S}_{64}$, have been considered. However, for the cases with highest $\mathrm{H}$ concentrations $\left(\mathrm{VH}_{4}\right)$, for which the defect-defect interaction might be important, also the $\mathrm{S}_{216}, \mathrm{~S}_{512}$ and $\mathrm{S}_{1000}$ supercells have been investigated. It is worth noting that $S_{1000}$ is by far the largest supercell for which the vibrational spectrum and the IR intensities have been computed.

Various spin states are considered $\left(\mathrm{S}_{z}=\frac{1}{2}\right.$ and $\frac{3}{2}$ for $\mathrm{VH}_{1} ; \mathrm{S}_{z}=1$ and 0 for $\mathrm{VH}_{2}$ and $\mathrm{V}$, the pure vacancy, here included for comparison; $\mathrm{S}_{z}=1 / 2$ for $\mathrm{VH}_{3}$ and $\mathrm{S}_{z}=0$ for $\mathrm{VH}_{4}$ ): their relative stability, band structure, charge and spin density distributions are analyzed. All the investigated systems present specific IR spectra, with peaks that can be used for the characterization of the defects. In particular the $\mathrm{C}-\mathrm{H}$ stretching peaks can be considered fingerprints of the system: they range (when anharmonicity is taken into account) from 2500 to $4400 \mathrm{~cm}^{-1}$ as a function of the number of $\mathrm{H}$ atoms in the vacancy. Anaharmonicity is extremely sensitive as well to the $\mathrm{H}$ load and spin state of the system, ranging from 340 $\mathrm{cm}^{-1}$ (the lowering of the wavenumber with respect to the harmonic value in $\mathrm{VH}_{1}$ ) to -84 $\mathrm{cm}^{-1}$ for $\mathrm{VH}_{4}$, where the anharmonic wavenumber increases with respect to the harmonic value due to the strong repulsion with the other $\mathrm{H}$ atoms. The graphical animation (see http://www.pmmp.jussieu.fr/yves/defectsindiamond/) permits to investigate the nature of the full set of modes.

The paper is structured as follows. In Section II information on the adopted method is pro- 
vided. Subsection II A is devoted to the vibrational frequencies computed in the harmonic approximation, and to the analytical estimation of the IR and Raman intensities. Subsection II B illustrates how the anharmonic contribution to the stretching is computed by solving numerically the corresponding one dimensional Schroedinger equation. Section III is organized in two parts. Subsection III A is devoted to the equilibrium geometry of the defect, to its charge distribution and to the band structure. In Subsection IIIB, the vibrational features of the defect are reported and analyzed, both at the harmonic and anharmonic level; comparison with experimental findings and previous simulations is performed. Finally, in Section IV some conclusions are drawn.

\section{COMPUTATIONAL MODELS AND DETAILS}

Calculations have been performed by use of the B3LYP global hybrid functional, ${ }^{11,12}$ as implemented in the CRYSTAL program. ${ }^{13}$ An all-electron basis set of Gaussian-type functions has been adopted (Pople's 6-21G) for carbon, whose exponent of the most diffuse sp shell is $0.23 \mathrm{Bohr}^{-2}$. For hydrogen, the basis set of the $6-31 \mathrm{G}$ set has been used. ${ }^{14}$ The defect formation energy and the $\mathrm{C}-\mathrm{H}$ anharmonic vibrational frequency have also been calculated with larger basis sets $\left(6-21 \mathrm{G}^{*}, 6-31 \mathrm{G}\right.$ and $\left.6-31 \mathrm{G}^{* 15}\right)$ and other functionals, ranging from pure $\left(\mathrm{LDA}^{16}\right.$ and $\left.\mathrm{PBE}^{17}\right)$, to global $\left(\mathrm{PBE} 0^{18}\right)$, and range-separated (HSE06 ${ }^{19}$ ) hybrids. The truncation of the Coulomb and exchange infinite lattice series is controlled by five thresholds $\mathrm{T}_{i}$ (see CRYSTAL manual, Ref. 20, for more details), which have been set to $8\left(\mathrm{~T}_{1}-\mathrm{T}_{4}\right)$ and $16\left(\mathrm{~T}_{5}\right)$. The convergence threshold on energy for the self-consistent-field $(\mathrm{SCF})$ procedure has been set to $10^{-8}$ Hartree for structural optimizations and to $10^{-10}$ Hartree for vibration frequency calculations.

The DFT exchange-correlation contribution to the Fock matrix has been evaluated by numerical integration over the unit cell volume. Radial and angular points for the integration grid are generated through Gauss-Legendre radial quadrature and Lebedev two-dimensional angular point distributions. The default pruned grid with 75 radial and 974 angular points has been used, whose accuracy can be measured by comparing the integrated charge density in the largest supercell here considered (128 atoms), $N_{i}=770.011$, with the total number of 770 electrons in the unit cell.

A periodic supercell approach is used to simulate different defect concentrations. Supercells 
containing 64 and 128 atoms have been considered (to be referred to in the following as $S_{n}$, with $n=64$ or 128 ). In the $\mathrm{V}_{4}$ case, for exploring the effect of larger dilutions of the defect on the IR spectrum, also the $S_{256}, S_{512}$ and $S_{1000}$ supercells have been considered. Reciprocal space has been sampled using a regular sub-lattice, centered at the origin (Гpoint), with a shrinking factor of 8 for $S_{64}, 4$ for $S_{128}$, and 2 for the larger supercells, which corresponds to a sampling over xx $\left(S_{64}\right)$ and $13\left(S_{128}\right)$ and zz $\left(S_{216}, S_{512}\right.$ and $\left.S_{1000}\right)$ kpoints in the irreducible part of the Brillouin zone for the case with highest symmetry $\left(\mathrm{VH}_{4}\right)$.

\section{A. Harmonic frequencies and the IR and Raman spectra}

Frequencies at the $\Gamma$ point are obtained within the harmonic approximation by diagonalising the mass-weighted Hessian matrix, $W$, whose elements are defined as ${ }^{21-25}$

$$
W_{\alpha i, \beta j}^{\Gamma}=\frac{H_{\alpha i, \beta j}^{\mathbf{0}}}{\sqrt{M_{\alpha} M_{\beta}}} \quad \text { with } \quad H_{\alpha i, \beta j}^{\mathbf{0}}=\left(\frac{\partial^{2} E}{\partial u_{\alpha i}^{\mathbf{0}} \partial u_{\beta j}^{\mathbf{0}}}\right),
$$

where $M_{\alpha}$ and $M_{\beta}$ are the masses of atoms associated with the $i$ and $j$ atomic coordinates. Once the Hessian matrix, $\mathcal{H}_{\alpha i, \beta j}$, has been calculated, frequency shifts due to isotopic substitutions can be calculated readily, at no computational cost, by changing masses in Eq. 1 . Energy first derivatives with respect to the atomic positions, $v_{\alpha, j}=\partial E / \partial u_{\alpha, j}$, are calculated analitically for all the $u_{\alpha, j}$ coordinates ( $E$ is the total energy, $u_{\alpha, j}$ is the displacement coordinate with respect to the equilibrium, $\alpha$ labels each atom), whereas second derivatives at $\vec{u}=0$ are calculated numerically using a single displacement along each coordinate $(N=2$, the central point and a point on the positive side):

$$
\left[\frac{\partial v_{\alpha j}}{\partial u_{\beta i}}\right] \approx \frac{v_{\alpha j}\left(0, \ldots, u_{\beta i}, \ldots\right)}{u_{\beta i}}
$$

or averaging two displacements $(N=3)$ :

$$
\left[\frac{\partial v_{\alpha j}}{\partial u_{\beta i}}\right] \approx \frac{v_{\alpha j}\left(0, \ldots, u_{\beta i}, \ldots\right)-v_{\alpha j}\left(0, \ldots,-u_{\beta i}, \ldots\right)}{2 u_{\beta i}}
$$

Previous calculations ${ }^{21,26,27}$ have shown that in bulk systems the influence of both $u$ and $N$ is very small (less than $1 \mathrm{~cm}^{-1}$ ) when $\mathrm{H}$ atoms are not present; much larger effects have been found for katoite ${ }^{28}$, brucite $\mathrm{e}^{29,30}$ and diaspore ${ }^{31}$, related to the anharmonicity of the $\mathrm{O}-\mathrm{H}$ stretching. More generally, anharmonicity is large in all cases in which $\mathrm{H}$ atoms are 


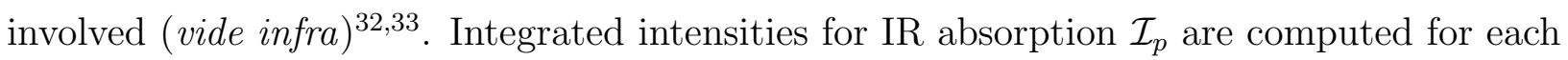
mode $p$ by means of the mass-weighted effective-mode Born-charge vector $\vec{Z}_{p}{ }^{34,35}$ evaluated through a CPHF/KS approach: ${ }^{36,37}$

$$
\mathcal{I}_{p} \propto\left|\vec{Z}_{p}\right|^{2}
$$

The relative Raman intensities of the peaks are computed analytically by exploiting a scheme illustrated in Refs. 38,39. Both schemes are based on the solutions of first- and second-order Coupled-Perturbed-Hartree-Fock/Kohn-Sham (CPHF/KS) equations. ${ }^{40,41}$

\section{B. The anharmonicity of the C-H stretching mode}

When both the fundamental frequency $\omega_{01}$ and the first overtone $\omega_{02}$ of a mode are available, the anharmonicity constant $\omega_{e} \chi_{e}$ can be obtained through the formula (see Ref. 30 for more details):

$$
\omega_{e} \chi_{e}=\frac{\left(2 \omega_{01}-\omega_{02}\right)}{2}
$$

that permits also the definition of the harmonic frequency $\omega_{e}$ as:

$$
\omega_{e}=\omega_{01}+2 \omega_{e} \chi_{e}
$$

The importance of anharmonicity in $\mathrm{H}$ containing systems is clear by looking at the anharmonicity constant $\omega_{e} \chi_{e}$ of some simple diatomic molecules such as $\mathrm{H}_{2}, \mathrm{HF}, \mathrm{HCl}$ and $\mathrm{HBr}$ where $\omega_{e} \chi_{e}$ is $121,90,53$ and $45 \mathrm{~cm}^{-1}$, respectively ${ }^{42}$. For the $\mathrm{O}-\mathrm{H}$ stretching, the anharmonicity can reach $200 \mathrm{~cm}^{-1}$, or even more when $\mathrm{O}-\mathrm{H}$ is involved in strong hydrogen bonds ${ }^{29,32}$. As regards the $\mathrm{C}-\mathrm{H}$ stretching, anharmonicity is estimated to be around 120-140 $\mathrm{cm}^{-1}$ for many organic molecules ${ }^{43,44}$.

The very large anharmonicity of the C-H, N-H and O-H stretching modes can be estimated taking advantage of their independence from the other normal modes. ${ }^{27-29,33}$

A scheme has been implemented in the CRYSTAL code that solves numerically the onedimensional Schrödinger equation when the potential energy, evaluated at 7 points along the $\mathrm{C}-\mathrm{H}$ coordinate, is fitted with a sixth order polynomial. ${ }^{45}$ The explored interval for the present case goes from -0.8 to $+1.0 \AA$ from the equilibrium position. The explored energy range is about $1.1 \mathrm{eV}$. The results vary by less than $1 \mathrm{~cm}^{-1}$ when a much richer set of points (26) is used in the same interval. 
Unfortunately this simple and effective scheme cannot be extended to the other vibrational modes involving hydrogen, such as bending, because in this case the separability with respect to the other modes is lost. In this case a full anharmonic treatment is required, that is not yet available in the CRYSTAL code.

\section{RESULTS}

Let us summarize the electronic situation of the systems we are considering in the present study. In the vacancy $\mathrm{V}$ there are 4 uncoupled electrons, that can generate three different states characterized by $\mathrm{S}_{z}=0,1$ and 2 (four spin up, three up and one down, two up and two down); we can call these states quintuplet, triplet and singlet, although only the first one is a pure spin state of the $\mathrm{S}^{2}$ operator. It should be underlined that the unpaired electrons remain localized to a large amount on the carbon atoms, as it should be, if and only if a large enough amount of "exact" Hartree-Fock (HF) exchange is included in the functional. With the HF method the localization is complete (one electron on each carbon atom); in "hybrid" functionals, such as B3LYP or PBE0 (very rarely adopted in previous studies for these systems), the localization remains large (about $0.8|e|$ on each dangling carbon bond, according to a Mulliken partition of the charge density). LDA and various versions of GGA, like PBE, are unable to localize these electrons, and the solution turns out, for many of the defects in diamond, to be metallic, in contrast with experimental evidence. The energy difference between the singlet and triplet states of the vacancy, evaluated at the B3LYP/6-21G level, is quite small $(0.13 \mathrm{eV})$, whereas the quintuplet is less stable by about $1.4 \mathrm{eV}^{46,47}$

The dangling bonds are progressively saturated by hydrogen atoms, so that in $\mathrm{VH}_{1}$ the three uncoupled electrons can generate a quadruplet $\left(q, \mathrm{~S}_{z}=3 / 2\right)$ or a doublet $\left(d, \mathrm{~S}_{z}=1 / 2\right)$; in $\mathrm{VH}_{2}$ the two unpaired electrons can generate a triplet $\left(t, \mathrm{~S}_{z}=1\right)$ or a singlet $\left(s, \mathrm{~S}_{z}=0\right)$. In $\mathrm{VH}_{3}$ the only unpaired electron is in a doublet state $\left(\mathrm{S}_{z}=1 / 2\right)$. Finally, in $\mathrm{VH}_{4}$ there are no uncoupled electrons and the ground state is a closed shell. The relative stability of the different spin states, and the stability of the various defects when the number of hydrogen atoms in the vacancy is increasing, are among the issues that will be discussed in this Section. 


\section{A. Geometry, charge and spin densities, band structure and the C-H homolytic}

dissociation

The geometry is relatively similar for the various cases here considered. As we are mainly interested in the behavior of the $\mathrm{C}-\mathrm{H}$ groups, we reported in Table I only two geometrical data, namely the $\mathrm{C}-\mathrm{H}$ and the $\mathrm{H}-\mathrm{H}$ distances (when applies). The former is equal to $1.07 \AA$ when a single $\mathrm{H}$ atom is present (in the low spin $\mathrm{VH}_{1}^{d}$ state), to $1.05 \AA$ when there are two $\mathrm{H}$ atoms in the cavity, to $1.03 \AA$ in the case with three $\mathrm{H}$ atoms, and to $1.01 \AA$ in $\mathrm{VH}_{4}$, as a consequence of the respulsion between hydrogen atoms. In the high spin $\mathrm{VH}_{1}^{q}$ state, with 3 spin up electrons, the $\mathrm{C}-\mathrm{H}$ distance is longer $(1.10 \AA)$, than in $\mathrm{VH}_{1}^{d}$ as a consequence of the strong Pauli repulsion due to the uncoupled electrons on the three carbon atoms.

The $\mathrm{H}-\mathrm{H}$ distance is $1.27 \AA$ in the cases with 2 and $3 \mathrm{H}$ atoms, and reduces to $1.25 \AA$ in $\mathrm{VH}_{4}$, due to the $\mathrm{HH}$ repulsion. The polarity of the $\mathrm{C}-\mathrm{H}$ bond is large when a single or two $\mathrm{H}$ atoms are present $\left(+0.17|e|\right.$ on $\mathrm{H}$ and -0.15 to $-0.13|e|$ on $\left.\mathrm{C}_{1}\right)$; it reduces progressively when the number of $\mathrm{H}$ atoms increases $\left(+0.12\right.$ and $-0.10|e|$ in $\mathrm{VH}_{3},+0.07$ and $-0.04|e|$ in $\left.\mathrm{VH}_{4}\right)$ so as to reduce the reciprocal repulsion among them. When the system is spin polarized, the spin density is essentially localized on the unsaturated $\mathrm{C}$ atoms (the magnetic moment of these $\mathrm{C}$ atoms is about $0.8|e|)$ : Table I shows that the amount of spin density on $\mathrm{H}$ and $\mathrm{C}_{1}$ is very small, the maximum being $+0.17|e|$ on $\mathrm{C}_{1}$ (a $\mathrm{C}$ atom linked to $\mathrm{H}$ ) for a system with three uncoupled electrons (the $\mathrm{VH}_{1}^{d}$ case); the magnetic moment of $\mathrm{H}$ is in all cases close to zero. The $\mathrm{C}-\mathrm{H}$ bond population increases from 0.27 to $0.31|e|$ from $\mathrm{VH}_{1}$ to $\mathrm{VH}_{3}$, in parallel to the shortening of the $\mathrm{C}-\mathrm{H}$ distance. $\mathrm{In}_{\mathrm{VH}_{4}}$, however, it slightly decreases. The $\mathrm{H}-\mathrm{H}$ bond population is negative (repulsive) and increases progressively up to $-0.10|e|$, a quite non negligible value, in $\mathrm{VH}_{4}$.

Bi-dimentional density maps, representing the Mulliken $\alpha$ - $\beta$ spin population, have been plotted for all open shell $S_{64}$ supercell systems in order to highlight the spin momentum density localized over the carbon atoms with the unpaired electrons. For all $\mathrm{VH}_{x}$ systems in $d, q, s$ and $t$ states the planes have been selected using symmetry criterion, involving the unsaturated carbon atoms. In quadruplet configuration of the $\mathrm{VH}_{1}$ system the three carbon atoms $\left(\mathrm{C}_{\mathrm{B}}, \mathrm{C}_{\mathrm{C}}, \mathrm{C}_{\mathrm{D}}\right.$ in Figure 1$)$ with their unpaired electron are symmmetry equivalent $\left(\mathrm{C}_{3 v}\right.$ point group) and the selected plane includes two of these unsaturated carbon and the $\mathrm{H}_{1}$ hydrogen atoms. For the doublet configuration $\left(\sigma_{v}\right.$ P.G. $)$ we have two carbon atoms $\left(\mathrm{C}_{\mathrm{B}}\right.$ 
and $\mathrm{C}_{\mathrm{C}}$ in Figure 1), symmetry equivalent, with same spin orientations and an other carbon $\left(\mathrm{C}_{\mathrm{D}}\right.$ in Figure 1$)$ with opposite spin momentum. In order to underline the spin pecularity of this system two different planes have been selected; one plane contains the $\mathrm{C}_{\mathrm{B}}, \mathrm{C}_{\mathrm{D}}$ (with opposite spin) and the $\mathrm{H}_{1}$ atoms, while the other plane includes the $\mathrm{C}_{\mathrm{B}}, \mathrm{C}_{\mathrm{C}}$ (with same spin) and the hydrogen $\mathrm{H}_{1}$. In the $\mathrm{VH}_{2}$ system in the triplet state $\left(C_{s}\right.$ point group) there are two unsaturated carbon atoms $\left(\mathrm{C}_{\mathrm{C}}\right.$ and $\mathrm{C}_{\mathrm{D}}$ in Figure 1$)$ with same spin configuarations, while in singlet state $\left(C_{1}\right.$ point group) that carbon atoms have an opposite spin. In both systems an identical plane has been choosen and it includes the $\mathrm{C}_{\mathrm{A}}, \mathrm{H}_{1}$ and $\mathrm{H}_{2}$ atoms and it corresponds to the $\sigma_{v}$ mirror plane defined in triplet configuration. In the last $\mathrm{VH}_{3}^{d}$ system $\left(\mathrm{C}_{3 v}\right)$ the plane selected contains $\mathrm{C}_{\mathrm{A}}, \mathrm{C}_{\mathrm{B}}$ and $\mathrm{H}_{1}$ atoms and it includes the unpaired electron localized on the $\mathrm{C}_{\mathrm{D}}$ carbon atom. The maps are shown in Figure 2 and in all cases the common aspect consists in the large localization of the spin density over the unsaturated carbon and an extented effect to the second atomic neighbours. The density polarization is more evident, in particular, for the quadruplet and triplet configurations: indeed this effect is due to the Pauli repulsion of the electrons involved in the adiacent unsaturated carbon atoms with same spin. In fact in the doublet and singlet states, for the alternation of $\alpha$ and $\beta$ electrons, the short range repulsions are rather reduced with a consequent energy lowering. The polarizition of spin density localized over the Hydrogen atoms is gradually decreased in the high spin configurations when the H-saturation increasing, while in low spin states is almost null (including the $\mathrm{VH}_{3}^{d}$ case).

The band structure of the systems and states here investigated are shown in Figure 3, where the energy levels associated to $\alpha$ and $\beta$ electrons are represented by continuous black and dotted red lines, respectively. All the investigated systems but $\mathrm{VH}_{4}$ have an open-shell ground state. The gap range of the explored configurations grows when the number of hydrogen increases, and it has an upper limit in the pristine diamond gap value $(5.76 \mathrm{eV})$. For $\mathrm{VH}_{4}$ it is $5.67 \mathrm{eV}$. In all other cases, defect bands related to the uncoupled electrons are present in the gap. In these cases the reference lowest difference between HOCO and LUCO are the singlet $(1.64 \mathrm{eV})$ and triplet states $(1.37 \mathrm{eV})$ of the pure vacancy. The number of the states situated in the diamond gap reduces as a consequence of hydrogen saturation, as the energy levels of the electrons in the band referred to a $\mathrm{C}-\mathrm{H}$ bond fall down in the occupied manifold.

In the $\mathrm{VH}_{1}$ case (three uncoupled electrons), the three energy levels appearing in the gap 
just below the Fermi energy (marked by the horizontal blue line) are two $\alpha$ and one $\beta$ for the doublet, and three $\alpha$ for the quadruplet. In $\mathrm{VH}_{2}$ (two uncoupled electrons), there are one $\alpha$ and one $\beta$ band under the Fermi levels for the singlet, and two $\alpha$ bands for the triplet. In $\mathrm{VH}_{3}$, there is a single uncoupled electron, with one $\alpha$ electron below the Fermi level. The energy dispersion of the levels occupied by the unpaired electrons is slightly smaller than $1.0 \mathrm{eV}$ for all spin states with the 64 atoms supercell, and is a measure of the interaction between defects belonging to different cells. When the supercells sizes increase, and then the defect concentrations reduces, the energy dispersion reduces too. ${ }^{10}$ In Figure 3 two different energy transitions are reported, both starting from the $\Gamma$-point. The first transition, which is a vertical one (turquoise arrow), involves the highest occupied level of the defect and the first unoccupied level of the diamond host. The second transition involves the last occupied and the first unoccupied energy levels of the defective states. These last transitions are reported with black and red arrows for $\alpha$ or $\beta$ transitions.

In Table II the energy required for the homolytic dissociation $\left(\mathrm{E}_{\mathrm{H}}\right)$ of the $\mathrm{VH}_{x}$ is reported, in agreement with the following reaction:

$$
\mathrm{VH}_{x} \leftrightharpoons \mathrm{V}^{s}+\frac{x}{2} \mathrm{H}_{2}
$$

The $s$ (singlet) superscript refers to the most stable state of the vacancy. These reactions, corresponding to the progressive saturation of the four dangling bonds in the vacancy, are always exothermic also when reference is done to half the energy of the $\mathrm{H}_{2}$ molecule $(-0.5844$ hartree). The energy would be higher by $2.31 \mathrm{eV}$ per $\mathrm{H}$ atom if reference would be done to atomic H (-0.4969 hartree, when the same basis set and functionals are used). Table II shows that, when the progressive insertion of hydrogen is considered, the energy gain is rapidly reducing, from $1.06 \mathrm{eV}$, then to $0.72 \mathrm{eV}(1.78-1.06)$ and to $0.33 \mathrm{eV}\left(2.11 \mathrm{eV}\right.$ for $\mathrm{VH}_{3}$ minus $1.78 \mathrm{eV}$ for $\mathrm{VH}_{2}^{s}$; the insertion of the last $\mathrm{H}$ atom is unfavorable by $0.02 \mathrm{eV}$ when reference is done to the $\mathrm{H}_{2}$ molecule (it remains however favorable by $2.29 \mathrm{eV}$ when the isolated $\mathrm{H}$ atom is used as a reference). The energy difference between the high and low spin states is $0.34 \mathrm{eV}$ for $\mathrm{VH}_{1}$, it reduces then to 0.10 for $\mathrm{VH}_{2}$; it is $0.13 \mathrm{eV}$ for the vacancy.

It is interesting to compare these numbers with the energy of similar reactions performed on molecules, when the same functional (B3LYP) and basis set (6-21G) are used. For ternary carbon atoms (a carbon linked to one $\mathrm{H}$ and three $\mathrm{C}$ atoms, as in isobutane) $\mathrm{E}_{\mathrm{H}}$ is $2.1 \mathrm{eV}$, then $1.05 \mathrm{eV}$ higher than for $\mathrm{VH}_{1}^{d}$. For secondary carbon atoms (the central carbon atom in 
propane, for example) it increases by about $0.2 \mathrm{eV}$, and the same step is observed in going from secondary to primary carbon atoms (in ethane, for example). It turns then out that the $\mathrm{C}-\mathrm{H}$ bond in $\mathrm{VH}_{1}$ is much weaker than typical $\mathrm{C}-\mathrm{H}$ bonds in molecules, and that the difference increases progressively when other $\mathrm{H}$ atoms are inserted in the vacancy. $\mathrm{E}_{\mathrm{H}}$ is as large as $1.7 \mathrm{eV}$ in $\mathrm{VN}_{3} \mathrm{H}^{48}$, larger then by $0.6 \mathrm{eV}$ than in $\mathrm{VH}_{1}$.

Table II reports, for comparison, also the defect formation energy $\left(\mathrm{E}_{\mathrm{f}}\right)$ obtained according to

$$
n \mathrm{C}+\frac{x}{2} \mathrm{H}_{2} \leftrightharpoons \mathrm{VH}_{x}
$$

where $n \mathrm{C}$ is a cell of pristine diamond containing $n$ carbon atoms. The trends along the series are obviously parallel to the ones for $\mathrm{E}_{\mathrm{H}}$; the formation energies turn out to the strongly hendothermic, due to the high stability of the starting compounds, ie diamond and $\mathrm{H}_{2}$ molecules.

\section{B. Spectroscopic characterization}

The IR and Raman spectra of pristine diamond are very simple: the former is completely flat, the latter is characterized by a single peak at $1332 \mathrm{~cm}^{-1}$ (the calculated one with the present functional and basis set is at $1317 \mathrm{~cm}^{-1}, 15 \mathrm{~cm}^{-1}$ below the experiment). The IR and Raman spectra of the pure vacancy V show some structure below the 1317 diamond Raman peak (two intense peaks at 1106 and $1301 \mathrm{~cm}^{-1}$ are the main features), whereas no peak appears above. ${ }^{46}$ All peaks in the high wavenumber interval (say $1332-5000 \mathrm{~cm}^{-1}$ ) must be thus attributed to the presence of hydrogen in our simulation (or possibly also to other kind of defects in experiments). We start our analysis from Figure 4, that shows the full IR spectra of the four systems (and six states) we are interested here. The spectra are obtained with the 64 atoms supercell; as in each panel the maximum is determined by a peak whose height can differ by a large amount from the others, a different scale is used in each panel. At the same time, in order to avoid the complete crush of many of the features of the spectra, the highest peak has been truncated properly, as indicated in the figures. The most striking feature emerging from the comparison of the six figure is the huge difference in the intensity scale, that can reach $232 \mathrm{~km} / \mathrm{mol}$ in $\mathrm{VNH}_{1}^{d}$ and $432 \mathrm{~km} / \mathrm{mol}$ in $\mathrm{VNH}_{1}^{q}$ (this state turns out however to be $0.32 \mathrm{eV}$ less stable than the doublet, so it is hardly observed in standard conditions). In $\mathrm{VH}_{2}^{s}$ the scale is two orders of magnitude smaller than in $\mathrm{VH}_{1}$, 
whereas in $\mathrm{VH}_{2}^{t}$ it is one order of magnitude larger. In $\mathrm{VH}_{3}^{s}$ the maximum is $90 \mathrm{~km} / \mathrm{mol}$, and in $\mathrm{VH}_{4}$ it is 5 times smaller.

If we focus however our attention on the bending and stretching modes that emerge from the pure diamond manifold (say modes above 1330-1350 $\mathrm{cm}^{-1}$ ), and can then be clearly identified, and disregard the excited spin states $\left(\mathrm{VH}_{1}^{q}\right.$ and $\left.\mathrm{VH}_{2}^{t}\right)$, the situation is the following (see also Table IV):

- In $\mathrm{VH}_{1}^{d}$ only one bending mode is visible, at $1421 \mathrm{~cm}^{-1}$ with intensity of $230 \mathrm{~km} / \mathrm{mol}$ (the intensity of the other bending mode at $1487 \mathrm{~cm}^{-1}$ is negligible, only $4 \mathrm{~km} / \mathrm{mol}$ ). The stretching mode at $3188 \mathrm{~cm}^{-1}$ is very intense $(935 \mathrm{~km} / \mathrm{mol})$.

- In $\mathrm{VH}_{2}^{s}$ there are two bending modes above $1350 \mathrm{~cm}^{-1}$, one at 1602 , the other at $1399 \mathrm{~cm}^{-1}$, the first with medium, the second with low intensity $(64$ and $26 \mathrm{~km} / \mathrm{mol}$, respectively). The two stretching modes at 3605 and $3404 \mathrm{~cm}^{-1}$ have very low intensity (9 and $16 \mathrm{~km} / \mathrm{mol})$.

- Also in $\mathrm{VH}_{3}^{d}$ there are two $\mathrm{C}-\mathrm{H}$ bending modes above $1350 \mathrm{~cm}^{-1}$, at 1530 and 1485 $\mathrm{cm}^{-1}$, both with medium-low intensity ( 55 and $32 \mathrm{~km} / \mathrm{mol}$ ). The symmetric stretching mode at 3909 has very low intensity $(33 \mathrm{~km} / \mathrm{mol})$; the antisymmetric one, at $3647 \mathrm{~cm}^{-1}$ is extremely intense $(1148 \mathrm{~km} / \mathrm{mol})$.

- In $\mathrm{VH}_{4}$ one bending mode at 1491 (three times degenerate) is very intense (224 $\mathrm{km} / \mathrm{mol}$ ); the other bending mode at $1408 \mathrm{~cm}^{-1}$, shown in Table IV and twofold degenerate, is silent due to symmetry together with the totally symmetric stretching at $4239 \mathrm{~cm}^{-1}$. The second one, at $3980 \mathrm{~cm}^{-1}$, has negligible intensity.

In a hypothetical diamond sample containing the four defects at the same concentration, thus, two peaks would dominate the bending region: the first one is at $1421\left(\mathrm{VH}_{1}^{d}\right)$, the second at $1491\left(\mathrm{VH}_{4}\right) \mathrm{cm}^{-1}$ and with about the same intensity.Also in the stretching region two peaks dominate: one at $3188\left(\right.$ again $\left.\mathrm{VH}_{1}^{d}\right)$ and one at $3647 \mathrm{~cm}^{-1}\left(\mathrm{VH}_{3}^{d}\right)$, with intensities that are 4 to 5 times larger than the largest one in the bending region. Looking at Figure 5 , top, in which this hypotetical spectrum in the high wavenumber region and referring to the $\mathrm{S}_{64}$ supercell is reported, it is evident that $\mathrm{VH}_{2}$ and $\mathrm{VH}_{4}$ are not visible in the stretching region.

Before leaving this part of the discussion, we can try to answer to the three following 
questions:

I) are there other bending modes related to $\mathrm{H}$ ?

II) are the wavenumbers here reported stable with respect to the defect dilution?

III) is the Raman spectrum giving more visibility to the systems that are essentially absent from the high wavenumber region of the IR spectrum, namely $\mathrm{VH}_{2}^{s}$ and $\mathrm{VH}_{4}$ ?

As regards the first question, in a sort of single particle approximation, that works reasonably well when hydrogen is involved, due to its small mass, one would expect three modes per $\mathrm{H}$ atom. Looking at Table IV, we discover that all the stretching modes are reported (the degeneracy should be taken into account), whereas a certain number of bending modes are lacking: 3 in $\mathrm{VH}_{4}$ and $\mathrm{VH}_{3}$, and two in the two spin states of $\mathrm{VH}_{2}$. Symmetry analysis, combined with the graphical animation of the modes, can help to identify the position of the "lacking" modes. For $\mathrm{VH}_{4}$ the threefold degenerate modes at $347 \mathrm{~cm}^{-1}$, of $\mathrm{F}_{1}$ symmetry, both IR and Raman inactive, complete the set of bending modes. For $\mathrm{VH}_{3}$ the twofold degenerate modes at $450 \mathrm{~cm}^{-1}$, of E symmetry, and the one at $442 \mathrm{~cm}^{-1}$, of $\mathrm{A}_{2}$ symmetry, complete the set. For $\mathrm{VH}_{2}^{s}$ the modes at 483 and $1352 \mathrm{~cm}^{-1}$, both of B" symmetry, complete the set.

As regards the second question, we open now a small discussion concerning the stability of the numbers just discussed when the size of the supercell is varying when the $\mathrm{VH}_{4}$ system is considered. The high wavenumbers part of the IR spectrum generated with the four cubic supercells $\left(\vec{a}=\vec{b}=\vec{c}\right.$ and $\left.\alpha=\beta=\gamma=90^{\circ}\right)$ containing $64,216,512,1000$ atoms are shown in Figure 8. The Figure permits to answer to two related questions, namely a) how is changing the IR spectrum with the defect concentration? and b) can we be sure that the differences are due to the different defect dilution and not, at least in part, to the different numerical errors accumulating for different sizes of the supercell?

As regards the second question, two evidences can be mentioned, referring to perfect systems, investigated with increasing supercells (in these cases obviously different supercells must provide the same frequencies and intensities): I) when the vibrational spectrum of perfect diamond is produced with supercells including an increasing number of atoms, from 2 to 1024, the Raman peak at 1317 moves by no more than $1 \mathrm{~cm}^{-1}$. II) Baima et al. ${ }^{25}$, in a paper devoted to the thermodynamics and phonon dispersion of pyrope and grossular, performed vibrational calculations with the CRYSTAL code on unit cells containing from 80 to 2160 atoms. The wavenumbers at the $\Gamma$ point show a maximum difference of only $0.357 \mathrm{~cm}^{-1}$ 
(Table 1 in the paper mentioned above). Figure 8 provides additional evidence for point a), and an answer to point $\mathrm{b}$ ). In short: the peak position in the $\mathrm{S}_{216}$ cell, differs by 1,3 and $8 \mathrm{~cm}^{-1}$ with respect to $\mathrm{S}_{1000}$; for $\mathrm{S}_{512}$ the difference reduces to 5,1 and $2 \mathrm{~cm}^{-1}$; also the intensities, that vary from 240 to $2 \mathrm{~km} / \mathrm{mol}$, are extremely stable from $\mathrm{S}_{216}$ on. We can conclude that the 3 indicated peaks still vary by some amount from $\mathrm{S}_{64}$ to larger cells as a consequence of the defect-defect interaction; from $\mathrm{S}_{216}$ on, the differences might also depend in part from negligibly small numerical noises of the code.

The answer to the last question can be found in Figure 7, where the high frequency part of the harmonic Raman spectrum is shown. The intensities provide a couple of surprises (the wavenumbers are obviously the same as for the IR spectrum): a) the symmetric $\mathrm{VH}_{4}$ peak has now non null intensity; b) all the seven peaks have intensities that differ by no more than $30 \%$, so that in the Raman spectrum all the $4 \mathrm{H}$ occupations should be visible at variance with respect to IR.

\section{The anharmonic treatment of the $\mathrm{CH}$ bending and stretching.}

Table V reports the results of the numerical solution of the one-dimensional Schrödinger equation along the $\mathrm{C}-\mathrm{H}$ coordinate, as described in Subsection II B. In the last two colums also the harmonic frequency $\omega_{H}$ obtained by diagonalizing the Hessian matrix, and using the one point $\omega_{H}^{1}$ or two points $\omega_{H}^{2}$ formulas (see equations 2 and 3) are reported. Data refer to the $\mathrm{S}_{64}$ supercell. The convergence with the supercell size, not shown, is as quick as for the harmonic frequency shown in Figure 8. The B3LYP fundamental anharmonic wavenumber of $\mathrm{VH}_{1}^{d}$ is at $3015 \mathrm{~cm}^{-1}, 217 \mathrm{~cm}^{-1}$ lower than the harmonic frequency $\left(\omega_{e}\right.$, at $3232 \mathrm{~cm}^{-1}$, see Equations 5 and 6 ). It is worth noting that the $\mathrm{C}-\mathrm{H}$ stretching of the $\mathrm{VN}_{3} \mathrm{H}$ defect ${ }^{48}$ is $120 \mathrm{~cm}^{-1}$ higher $\left(3135 \mathrm{~cm}^{-1}\right.$ at the same level of theory), whereas the one of the ternary carbon of isobutane (the $\mathrm{C}$ atom is linked to one $\mathrm{H}$ and three $\mathrm{C}$ atoms, a local situation similar to the one of $\left.\mathrm{VH}_{1}\right)$ is about $150 \mathrm{~cm}^{-1}$ lower $\left(2862 \mathrm{~cm}^{-1}\right)$. The anharmonicity of $\mathrm{VH}_{1}^{q}$ is much larger than the $d$ one: 335 vs $217 \mathrm{~cm}^{-1}$ (in $\mathrm{VN}_{3} \mathrm{H}$ and isobutene it is much smaller: 138 and $116 \mathrm{~cm}^{-1}$, respectively ${ }^{48}$ ). The difference between $\omega_{e}$ and $\omega_{H}$ (last column) is not surprising: when the energy surface is very anaharmonic, as in the present case, the use of only two energy points (the equilibrium and one displaced to the right or to the left, as for $\omega_{H}$ ) is not enough for an accurate estimate of the second 
derivative in the minimum.

Frequency analysis of the four saturations:

All six studied systems have been approached calculating the anharmonic approximation and successively compared with the respective harmonic frequencies in order to obtain anharmonicity estimation. The anharmonic contributions have been evaluated as a function of the C-H stretching, resolving the one-dimensional Schrödinger equation. In this approach the carbon backbone of the cell is frozen, leaving free to move only the hydrogen atoms. Obviously this kind of anharmonic approximation provides a reasonable estimation only for the strenghing modes; in fact the stretching are strongly decorrelated respect to the other modes and stretching-bending coupling can be neglected introducing a variable error in the anharmonic evaluation. When more then one Hydrogen atoms is prensent, it is worth of note that the symmetric and anti-symmetric anharmonicity can be evaluated keeping or breaking the symmetry in $\mathrm{C}-\mathrm{H}$ displacement; indeed in the former case the hydrogen atoms are moved simultaneously, reproducing a symmetrical behaviour, while in the latter case hydrogen atoms are moved one at a time, miming an anti-symmetric vibrations. The entity of the anharmonic shift is different in each cases: from 1 to 2 hydrogen atoms saturating the vacancy, we obtain a red shift.Whereas when there are 3 or 4 hydrogen atoms, saturating the vacancy, a blue shift has been observed. Usually the anharmonic contribution is more pronounced for the high spin state (quadruplet and triplet state), and in each case the symmetrical stretching have a bigger shift if is compared to the anti-symmetric. In $\mathrm{VH}_{1}$ system, considering the anharmonic shift of the stretching modes, it can be found one vibration at $2797 \mathrm{~cm}^{-1}$ with an intensity of $82 \mathrm{~km} / \mathrm{mol}$ for the doublet state and one at $2483 \mathrm{~cm}^{-1}$ with $256 \mathrm{~km} / \mathrm{mol}$ intensity for the quadruplet state. The energy difference between the two states is $0.13 \mathrm{eV}$ (Table II). The corresponding Boltzman population ratio between the quadruplet and doublet state at $298.15 \mathrm{~K}$ is 0.00634 . This is roughly estimation because the dft-energy difference is computed at $0 \mathrm{~K}$ and probably the effect of the temperature will populate the high spin state, increasing the Boltzmann ratio. These peaks represent the most intensive features in the IR spectrum. In attempt to match this feature with experimental work of Fritsh $^{4}$ we can explore the literature data in a range of $/ p m 20 \mathrm{~cm}-1 \mathrm{~cm}^{-1}$ respect to the theoretical values. Different signal follow in this frequencies range for the doublet state: the $2784(6)$ and $2798 \mathrm{~cm}^{-1}$ peaks attributed to the $\mathrm{H}$ in diamond type $\mathrm{Ib}^{2}$ and the $2812 \mathrm{~cm}^{-1}$ 
peak referred to hydrogen in cuboid sector of historical asteriated hydrogen-rich diamonds ${ }^{49}$. For the doublet it cannot find any signal; indeed in the range 1461-2784, in the experimental data, there is an empty region. In addition, the strong intensity of this peak, would make it clearly visible in the spectrum and since there is no trace, this aspect could be considered as an evidence of the absence of this defect. For the $\mathrm{VH}_{2}$ system the difference in energy between the two spin states is $0.34 \mathrm{eV}$, this larger energy gap make the triplet state unpopulated (Boltzmann ratio of $1.810^{-6}$ ), and so we have only considered the more stable state. This defect has a maximum intensity lower then the one hydrogen saturation case $(1-7 \mathrm{~km} / \mathrm{mol})$. The symmetrical stretching of the low spin state, in anharmonic approximation, have one feature at $3552 \mathrm{~cm}^{-1}$. The effect of anharmonic correction is stronger then the system with only one hydrogen, due to the steric repulsion of the hydrogen atoms in the vacancy cavity and in attempt to find a signal matching between the experiments and theoretical calculation the range of experimental frequencies centred to the theoretical spot must be enlarged. In a wider range of $/ p m 50 \mathrm{~cm}^{-1}$ it is possible to find a signal at 3525 releted to (L. Massi:Etude des dfauts dans les diamants bruns et les diamants riches en hydrogne(PhD thesis, University of Nantes, France, 2006e)). The asymmetrical stretching shows one theoretical feature at $3378 \mathrm{~cm}^{-1}$. This spot can be matched with one experimental features in $/ p m 50 \mathrm{~cm}^{-1}$ range; indeed there are $3354 \mathrm{~cm}^{-1}$ signal refereed to [L. Massi: Etude des dfauts dans les diamants bruns et les diamants riches en hydrogne $(\mathrm{PhD}$ thesis, University of Nantes, France, 2006) (in French) In the $\mathrm{VH}_{3}$ system the anharmonic symmetrical stretching $\left(3943 \mathrm{~cm}^{-1}\right)$ and anti-symmetric $\left(3653 \mathrm{~cm}^{-1}\right)$ stretching dont have any matching with experimental data; indeed in the experimental data there is a not populated region going from 3525 to $4167 \mathrm{~cm}^{-1}$. The $\mathrm{VH}_{4}$ system has the symmetrical stretching IR inactive for symmetry and the anti-symmetric follow at $4037 \mathrm{~cm}^{-1}$, and it falls into the same vacuum region as described above.

\section{CONCLUSIONS}

\section{COPIA}

In this work a series of defects consisting of hydrogen atoms populating the vacancy in diamond have been investigated $\left(\mathrm{VH}_{y}, \mathrm{y}=1\right.$ to 4$)$. Only the highest loading $\operatorname{defect}\left(\mathrm{VH}_{4}\right)$ has a closed shell ground state. $\mathrm{VH}_{1}$ can exist in the quadruplet and doublet spin states (three 
uncoupled electrons), $\mathrm{VH}_{2}$ in a triplet and singlet state, $\mathrm{VH}_{3}$ only in the doublet state.

Two main issues have been considered in the present study:

i) are all the model here proposed realistic from the energetic point of view? Can they be inserted in the long list of defects in diamond?

ii) if they exist, are they also visible from the experimental point of view, in particular do they produce visible, intense IR peaks?

The answer to the first question is positive. If reference is done to the $\mathrm{V}$ defect, then the saturation with hydrogen of one of the carbon atoms surrounding the vacancy is an exothermic process, also in the less favourable case in which the $\mathrm{H}$ atom comes from $\mathrm{H}_{2}$ and then requires to brake a strong covalent bond. The bonds with neighboring $\mathrm{C}$ atoms of the many $\mathrm{H}$ atoms floating around in diamond (for example in interstitial positions) is weaker than in $\mathrm{H}_{2}$, and then the hydrogenation of $\mathrm{V}$ becomes more favourable.

As regards the second question, we focused here our attention on the $\mathrm{CH}$ stretching modes, that appear at wavenumbers much higher than $1340 \mathrm{~cm}^{-1}$, and then far away from the Diamond $+\mathrm{V}+\mathrm{N}$ manyfold, characterized by a superposition of vibrational bands that form essentially a continuum. The 10 stretching modes characterizing the systems here investigated span nearly $1000 \mathrm{~cm}^{-1}$, and should be in principle detectable individually, so as to permit to identify unambiguously all these defects. However most of them do have a quite low intensity, so that they are hardly visible in the very rich IR experimental spectrum, to which many other kind of defects are probably contributing. The only one easily detectable in experiments is the peak at $3107 \mathrm{~cm}^{-1}$, that has an intensity of $621 \mathrm{~km} / \mathrm{mol}$, and is now attributed unambiguously to the $\mathrm{VN}_{3} \mathrm{H}$ defect. The second most intense peak has intensity of $346 \mathrm{~km} / \mathrm{mol}$ (just a bit more than $50 \%$ the one of $\mathrm{VN}_{3} \mathrm{H}$ ), whereas the other 8 peaks span from $30 \%$ (just one) of it, to nearly null intensity. It should be noticed that other defects can have quite high intensity, and then generate large bands that completely hidden the small peaks. For exmaple the stretching mode of VNH, whose anharmonic wavenumber is at $\mathrm{xx}$, has intensity of $900 \mathrm{~km} / \mathrm{mol}$, then $50 \%$ higher than the more intense one of this set (we remind that the IR intensity can be evaluated in an absolute scale, so that cross comparison between different systems is possible also ina bsolute values. As a consequence of the previous discussion,we can conclude that many of the defects here investigated (actually, 
ALL of them) can exists in defective diamond, but the evidence of their existence cannot be obtained for most of them from IR spectra. 
Figures 


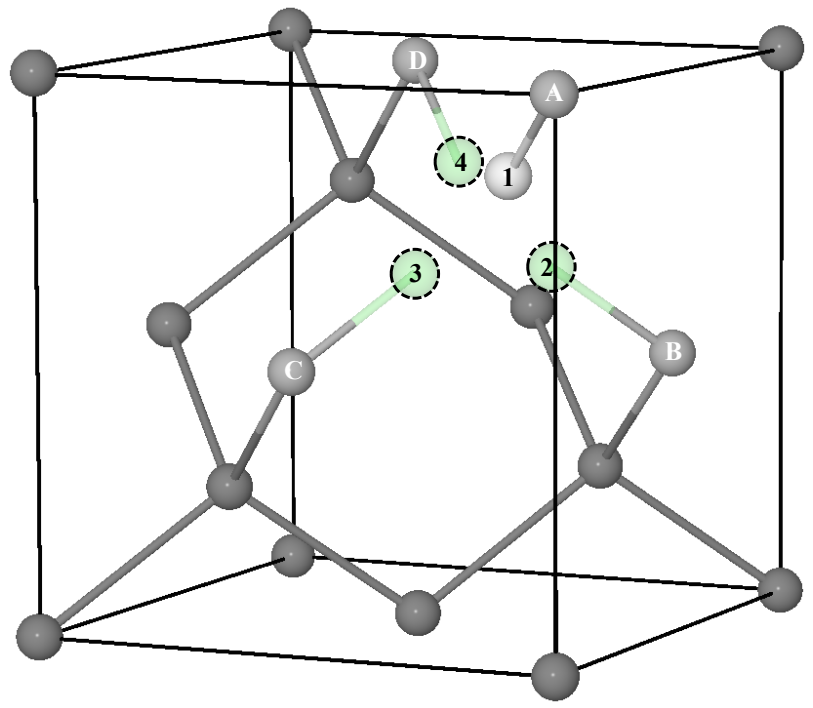

FIG. 1: A schematic representation of the $\mathrm{VH}_{1+n}(n=0,1,2$ and 3$)$ defects. Green positions can be either empty $\left(\mathrm{VH}_{1}\right)$ or occupied by hydrogen atoms (position 2 in $\mathrm{VH}_{2}$, position 2 and 3 in $\mathrm{VH}_{3} ; 2,3$ and 4 in $\left.\mathrm{VH}_{4}\right)$. 

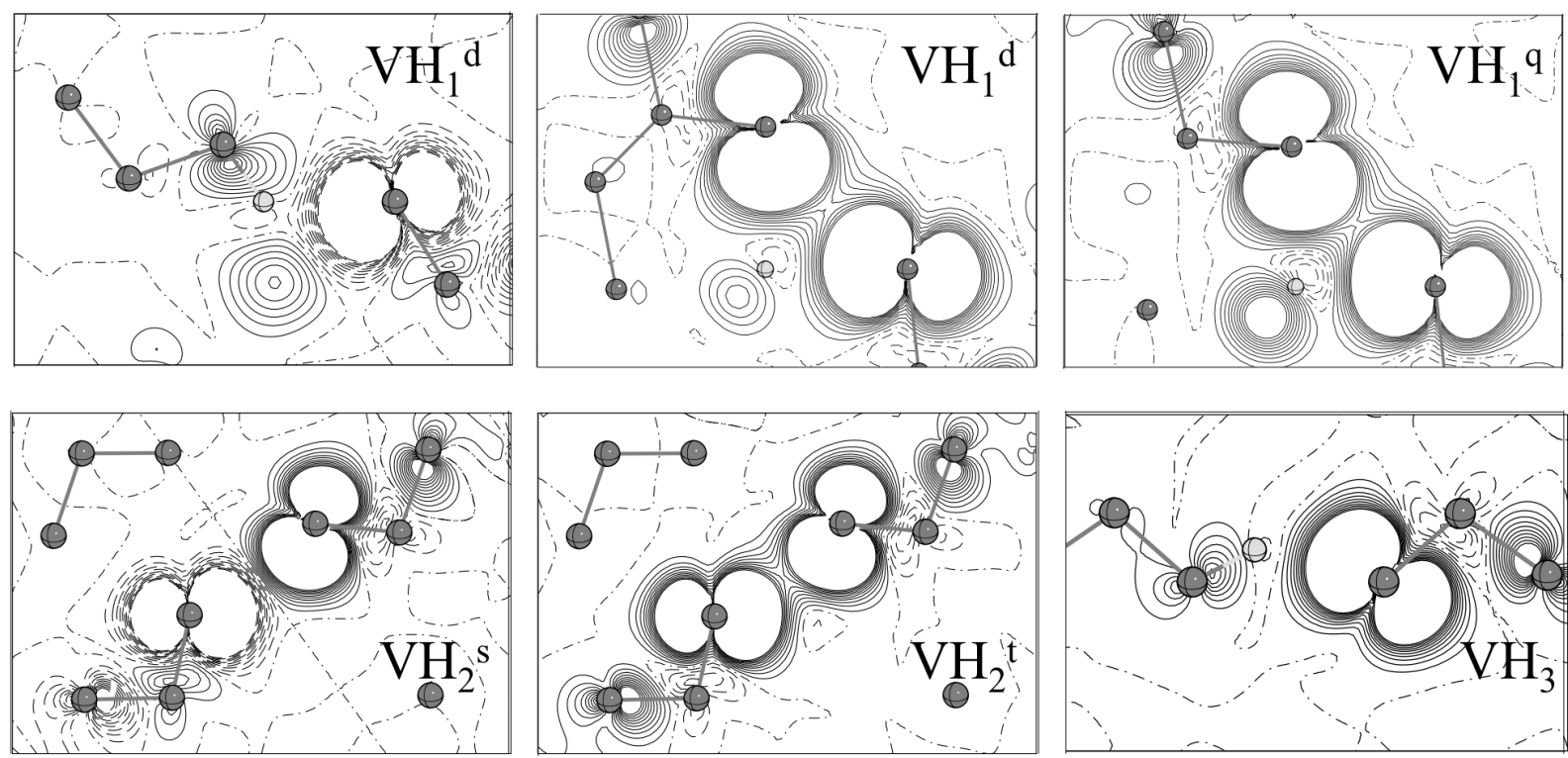

FIG. 2: Spin density maps of the $\mathrm{VH}_{1}^{d}$ (projected over two differents planes), $\mathrm{VH}_{1}^{q}, \mathrm{VH}_{2}^{s}, \mathrm{VH}_{2}^{t}$ and $\mathrm{VH}_{3}$ defects. The three points defining the planes, in the case of $\mathrm{VH}_{1}^{d}$ system the plane in the first figure contains the carbon atoms with opposite spin orientations respect to the other two carbons. To the contrary in the second figure the plane contains the two carbon with same spin configuration. In the case of $\mathrm{VH}_{2}$ system, are the two $\mathrm{C}$ and $\mathrm{D}$ (carbon atoms with the unpaired electrons) with the centre of the vacancy. They are represented in bottom panel. In the $\mathrm{VH}_{3}^{d}$ system (last immage in bottom panel) the plane has been defined by the $\mathrm{D}$ atom (the only carbon atom with an unpaired electron), hydrogen $\mathrm{H}_{1}$ and carbon D. See Figure 1 for the labels of the atoms. Isodensity lines differ by $0.01|\mathrm{e}| /\left(a_{0}\right)^{3}$; spin density is truncated at $\pm 0.1|\mathrm{e}| /\left(a_{0}\right)^{3}$. Continuous, dashed and dot-dashed lines indicate positive, negative and zero values, respectively. 

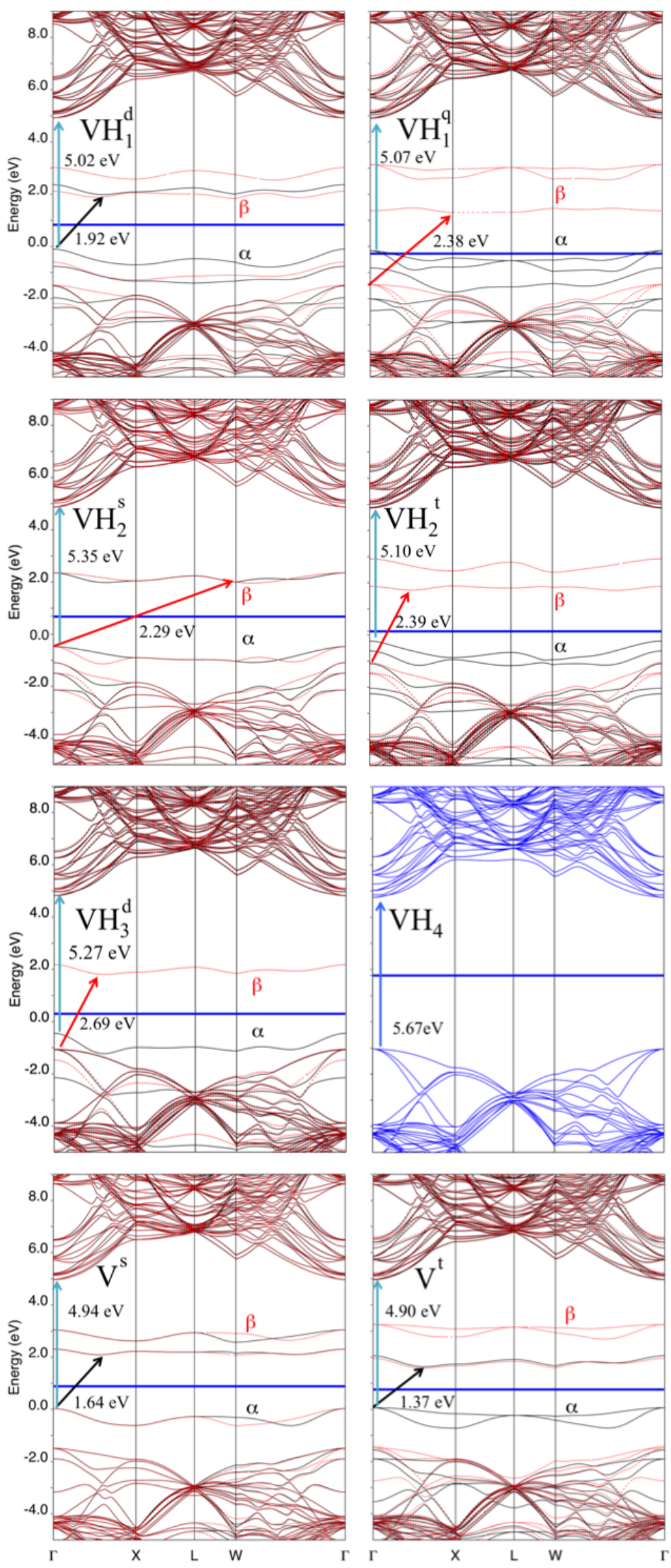
FIG. 3: Electronic band structure of the diamond vacancy with incremental hydrogen saturation. The top panels refer to the $\mathrm{VH}_{1}$ quadruplet $q$ (left) and doublet $d$ (right) electronic states. The second row panels show the $\mathrm{VH}_{2}$ triplet $t$ (left) and singlet $s$ (right) band structure. The third row panels refer to $\mathrm{VH}_{3}$ in its $d$ state, whereas $\mathrm{VH}_{4}$, in its closed shell ground state, is shown to the right. The vacancy $\mathrm{V}^{t}$ (left) and $\mathrm{V}^{s}$ (right) states are also shown, for completeness, in the bottom panels. Black and red bands correspond to $\alpha$ and $\beta$ electrons, respectively. The blue line is the Fermi level. Data obtained with the $\mathrm{S}_{64}$ supercell. 

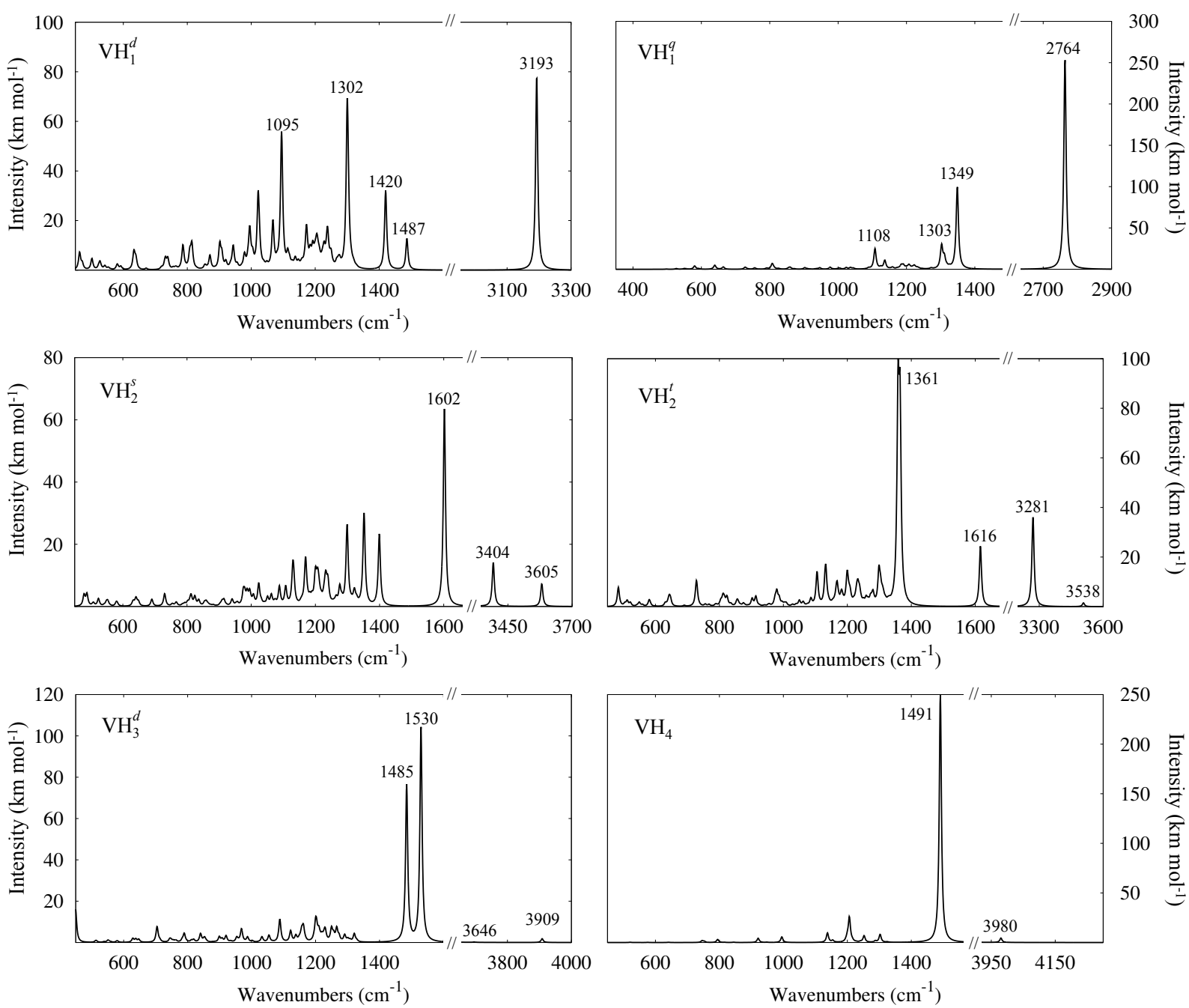

FIG. 4: Infrared spectra of the four $\mathrm{VH}_{n}(n=1$ to 4$)$ defects, in their various spin states, computed with the $\mathrm{S}_{64}$ supercell. 


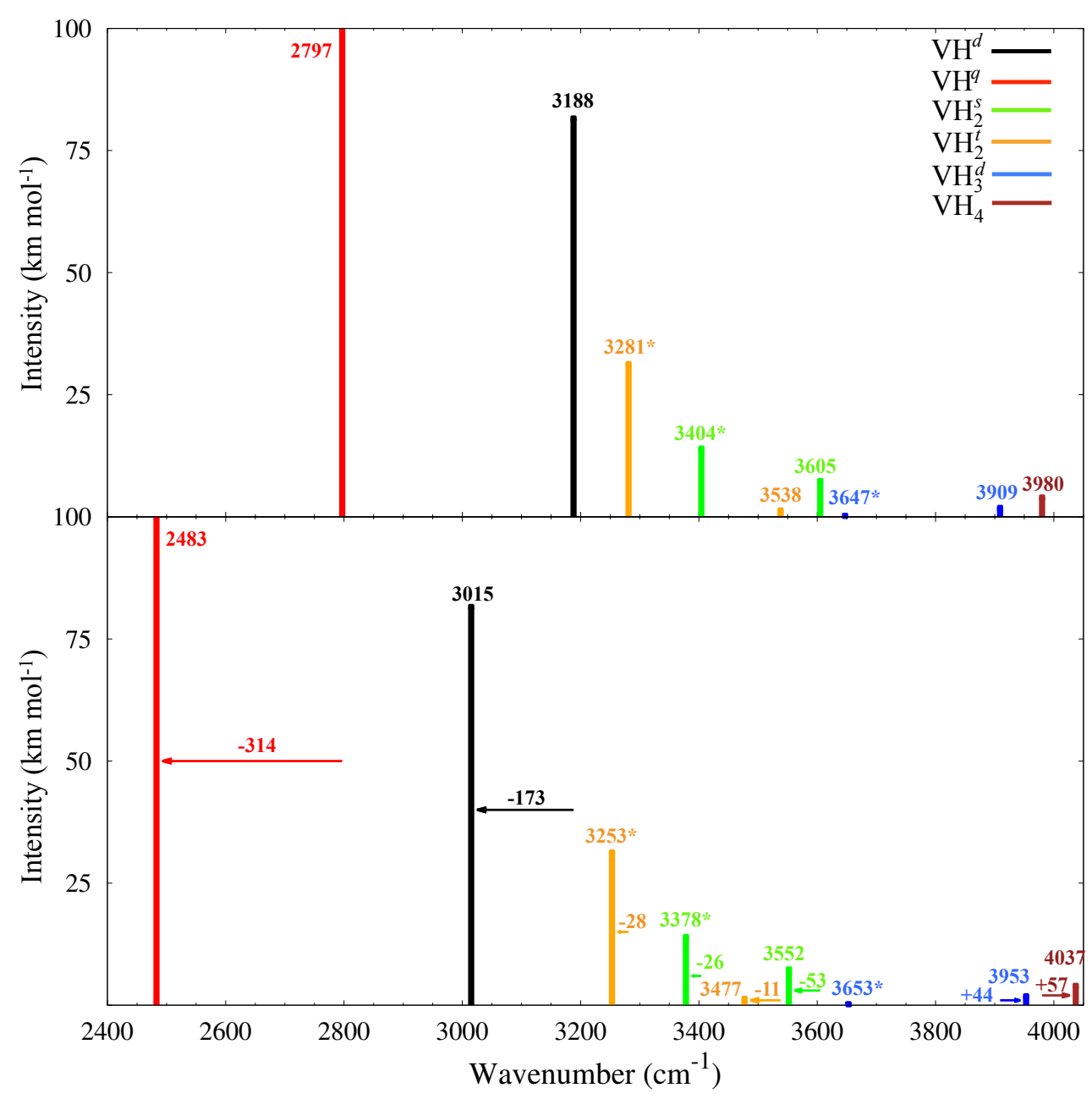

FIG. 5: Harmonic (top) and anharmonic (bottom) wavenumbers for the $\mathrm{C}-\mathrm{H}$ stretching mode(s) for the $\mathrm{VH}_{x}$ defects. When more than one hydrogen atom is present there are two stretching modes (symmetric, at high wavenumber, and antisymmetric, at low wavenumber, and marked with an asterisk). The height of each bar is the simulated IR intensity evaluated at the harmonic level. Calculations refer to B3LYP/6-21G, and to the $\mathrm{S}_{64}$ supercell. 


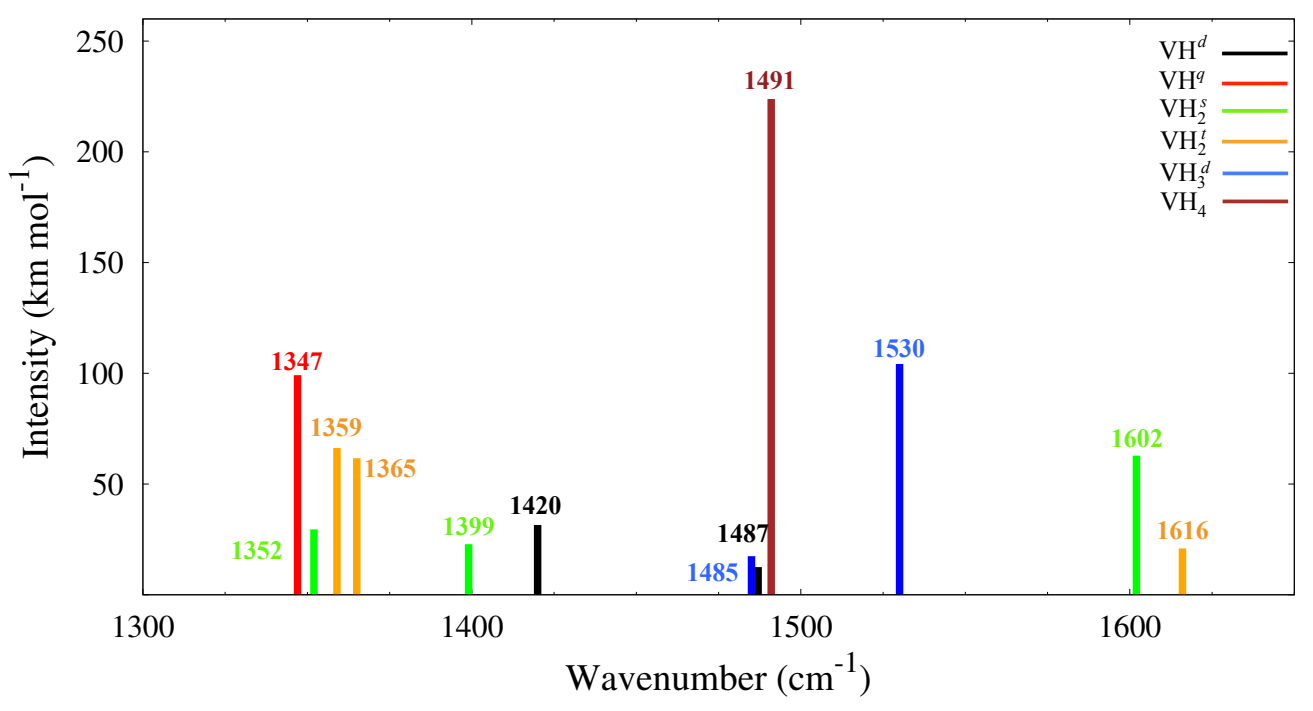

FIG. 6: Harmonic wavenumbers for the $\mathrm{C}-\mathrm{H}$ bending modes for the $\mathrm{VH}_{x}$ defects. The height of each bar is the simulated IR intensity evaluated at the harmonic level. Calculations refer to B3LYP/6-21G, and to the $\mathrm{S}_{64}$ supercell. 

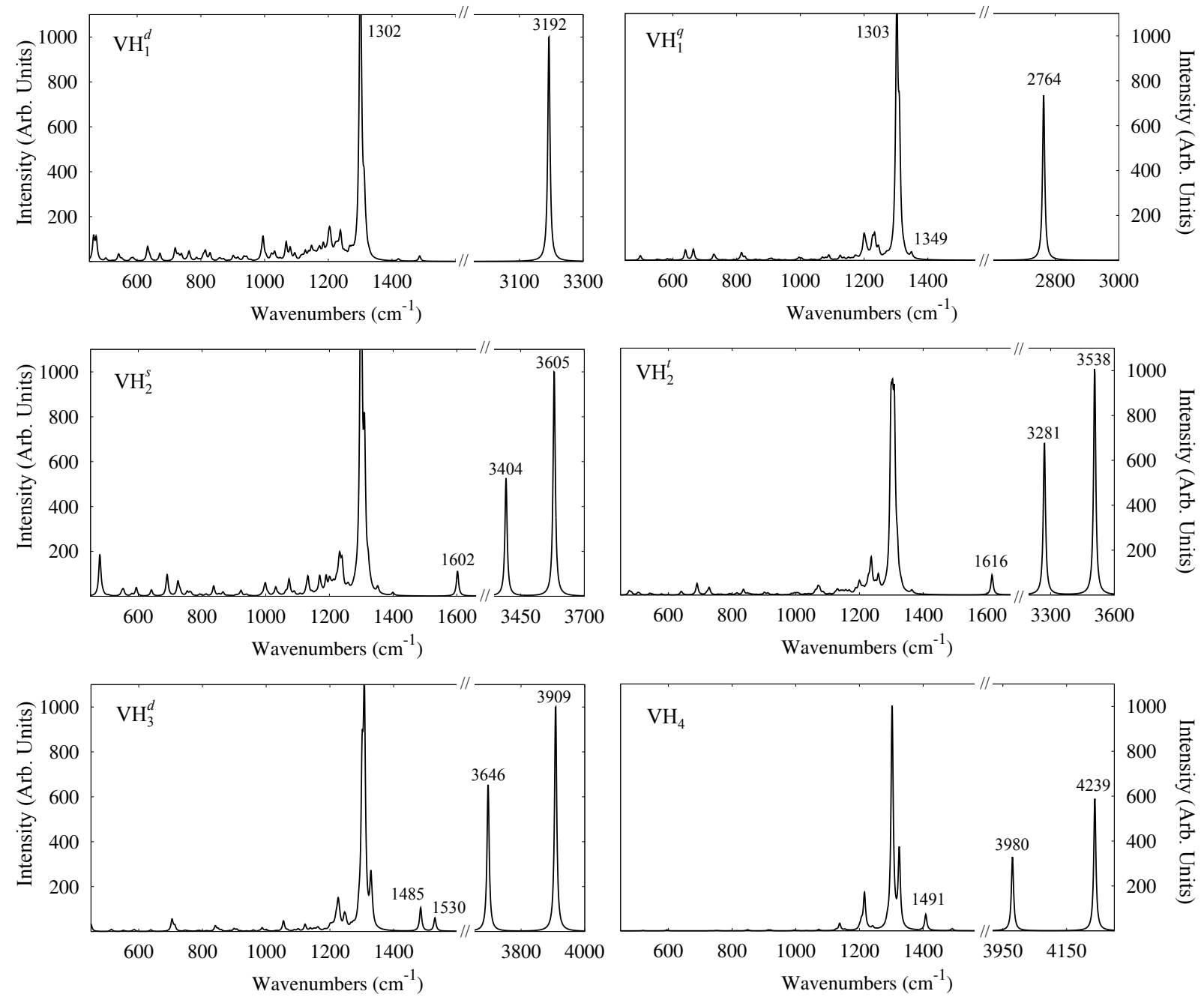

FIG. 7: Simulated Raman spectra of the four $\mathrm{VH}_{n}(n=1$ to 4$)$ defects, in their various spin states, computed with the $\mathrm{S}_{64}$ supercell. 


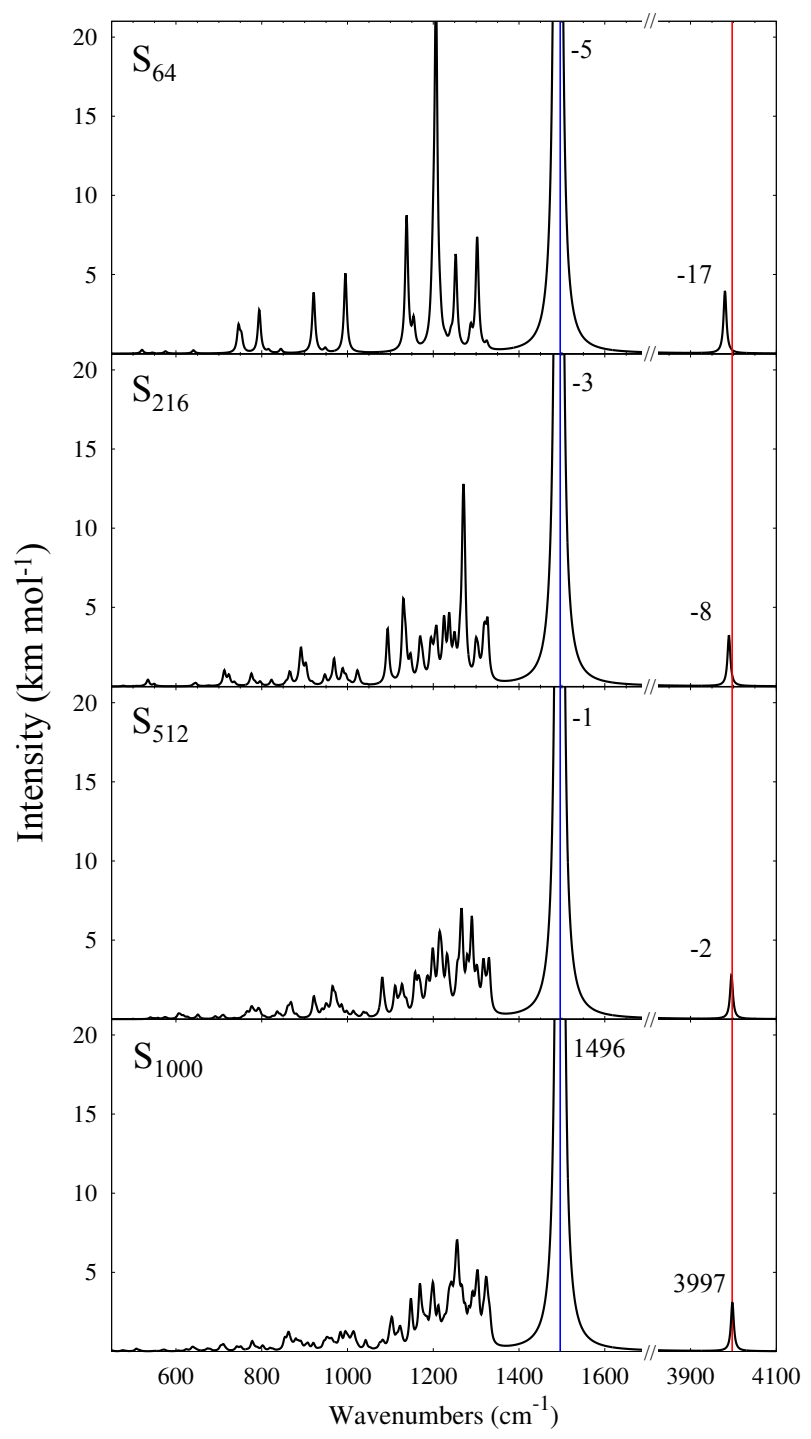

FIG. 8: The effect of the defects dilution on the computed IR spectrum of $\mathrm{VH}_{4}$. Four supercells containing 64, 216, 512 and 1000 atoms are considered. Vertical blue and red lines mark the position of hydrogen bending and asymmetric stretching modes (both triply degenerate), respectively, for the $\mathrm{S}_{1000}$ case. Concerning $\mathrm{S}_{64}, \mathrm{~S}_{216}$, and $\mathrm{S}_{512}$ cases, frequency shifts with respect to the $\mathrm{S}_{1000}$ (bottom figure) are reported. 
Tables 


\begin{tabular}{cccccccccc}
\hline \hline System & $\mathrm{R}_{C H}$ & $\mathrm{R}_{H H}$ & $\mathrm{Q}_{H}$ & $\mathrm{Q}_{C 1}$ & $\mu_{H}$ & $\mu_{C 1}$ & $\mathrm{~B}_{C H}$ & $\mathrm{~B}_{H H}$ \\
\hline $\mathrm{VH}_{1}^{d}$ & 1.072 & - & +0.17 & -0.15 & -0.01 & +0.04 & +0.27 & - \\
$\mathrm{VH}_{1}^{q}$ & 1.108 & - & +0.18 & -0.13 & -0.02 & +0.17 & +0.26 & - \\
\hline $\mathrm{VH}_{2}^{s}$ & 1.052 & 1.273 & +0.17 & -0.14 & 0.00 & 0.00 & +0.30 & -0.04 \\
$\mathrm{VH}_{2}^{t}$ & 1.059 & 1.277 & +0.17 & -0.13 & -0.01 & +0.07 & +0.30 & -0.04 \\
\hline $\mathrm{VH}_{3}^{d}$ & 1.036 & 1.273 & +0.12 & -0.10 & -0.00 & +0.03 & +0.31 & -0.07 \\
\hline $\mathrm{VH}_{4}$ & 1.017 & 1.251 & +0.07 & -0.04 & - & - & +0.29 & -0.10 \\
\hline \hline
\end{tabular}

TABLE I: Structural and Mulliken population data for the $\mathrm{VH}_{x}^{y}$ defects, where the $\mathrm{y}$ superscipt can be $s$ (singlet), $d$ (doublet), $t$ (triplet) and $q$ (quadruplet). In the case of closed shell states, the y superscript is absent. $\mathrm{R}_{C H}$ and $\mathrm{R}_{H H}$ are the shortest distances (in $\AA$ ) between the indicated atoms, $\mathrm{B}_{C H}$ and $\mathrm{B}_{H H}$ are the corresponding bond populations, $\mathrm{Q}_{X}$ and $\mu_{X}$ are the net electronic charges and magnetic moments of atom $X\left(\mathrm{~B}, \mathrm{Q}\right.$ and $\mu$ in $|e|$ units). All data refer to the $\mathrm{S}_{64}$ supercell. 


\begin{tabular}{cccccc}
\hline \hline & $\mathrm{E}_{\mathrm{VH}_{x}}$ & $\mathrm{E}_{\mathrm{H}}$ & $\mathrm{E}_{f}$ & $\delta \mathrm{E}$ \\
$\mathrm{V}^{s}$ & -2397.2713 & \multicolumn{2}{c}{-6.85} & \\
& & & & 0.13 \\
$\mathrm{~V}^{t}$ & -2397.2664 & & & 0.0 \\
\hline $\mathrm{VH}^{d}$ & -2397.8945 & 1.06 & -5.79 & \\
$\mathrm{VH}^{q}$ & -2397.8820 & 0.72 & -6.13 & 0.34 \\
\hline $\mathrm{VH}_{2}^{s}$ & -2398.5054 & 1.78 & -5.07 & \\
$\mathrm{VH}_{2}^{t}$ & -2398.5015 & 1.67 & -5.18 & 0.10 \\
\hline $\mathrm{VH}_{3}^{d}$ & -2399.1019 & 2.11 & -4.74 & \\
\hline $\mathrm{VH}_{4}$ & -2399.6855 & 2.09 & -4.76 & \\
\hline \hline
\end{tabular}

TABLE II: Homolytic dehydrogenation $\left(\mathrm{E}_{\mathrm{H}}\right.$, in $\left.\mathrm{eV}\right)$ and formation $\left(\mathrm{E}_{f}\right.$, in $\left.\mathrm{eV}\right)$ energies of $\mathrm{VH}_{x}$ evaluated according to equations 7 and 8 , respectively. $\delta \mathrm{E}$ (in $\mathrm{eV}$ ) is the energy difference between the highest and lowest spin state (the former is more stable in all cases). The total energy of the various systems, in hartree, is also shown in the first column. The H energy, -0.5844 hartree, is half the energy of the $\mathrm{H}_{2}$ molecule. The energy of the isolated $\mathrm{H}$ atom is $0.4969 \mathrm{eV}$. The pure diamond energy is -2435.58 hartree. Data refer to the $\mathrm{S}_{64}$ supercell. 


\begin{tabular}{lcccccccc}
\hline \hline Method & $\mathrm{S}_{x}$ & $\mathrm{E}_{\mathrm{H}_{2}}$ & $\mathrm{E}_{\mathrm{C}}^{64}$ & $\mathrm{E}_{\mathrm{V}^{s}}$ & $\mathrm{E}_{\mathrm{VH}_{4}}$ & $\mathrm{E}_{G}$ & $\mathrm{E}_{\mathrm{H}}$ & $\mathrm{E}_{f}$ \\
\hline \hline B3LYP & $\mathrm{S}_{64}$ & -1.1687 & -2435.5789 & -2397.2713 & -2399.6855 & 5.67 & 2.09 & 4.76 \\
B3LYP & $\mathrm{S}_{128}$ & -1.1687 & -4871.1578 & -4832.8486 & -4835.2591 & 5.71 & 1.99 & 4.90 \\
\hline HF & $\mathrm{S}_{64}$ & -1.1268 & -2421.7608 & -2383.6978 & -2385.9704 & 11.79 & 0.51 & 5.55 \\
PBE0 & $\mathrm{S}_{64}$ & -1.1639 & -2434.8068 & -2396.4979 & -2398.9123 & 5.77 & 2.36 & 4.86 \\
HSE06 & $\mathrm{S}_{64}$ & -1.1636 & -2434.7622 & -2396.4556 & -2398.8676 & 5.13 & 2.31 & 4.86 \\
PBE & $\mathrm{S}_{64}$ & -1.1620 & -2434.4882 & -2396.2049 & -2398.6029 & 3.97 & 2.02 & 4.64 \\
LDA & $\mathrm{S}_{64}$ & -1.1333 & -2416.0004 & -2377.9867 & -2380.5056 & 3.96 & 6.87 & 0.31 \\
\hline \hline
\end{tabular}

TABLE III: Total (in hartree), homolytic dehydrogenation $\left(\mathrm{E}_{\mathrm{H}}\right.$, in $\left.\mathrm{eV}\right)$ and formation $\left(\mathrm{E}_{f}\right.$, in eV) energies of $\mathrm{VH}_{4}$ obtained with various methods. $\mathrm{E}_{\mathrm{V}^{s}}$ is the energy of the vacancy in the singlet state. $\mathrm{E}_{\mathrm{H}}$ and $\mathrm{E}_{f}$ are obtained according to Equations 7 and 8, respectively. $\mathrm{E}_{G}$ is the band gap. 


\begin{tabular}{cccccccccc}
\hline \hline \multicolumn{3}{c}{ Stretching } & \multicolumn{5}{c}{ Bending } \\
& $\omega_{\mathrm{S}_{1}}$ & $\mathrm{I}_{\mathrm{S}_{1}}$ & $\omega_{\mathrm{S}_{2}}$ & $\mathrm{I}_{\mathrm{S}_{2}}$ & $\omega_{\mathrm{B}_{1}}$ & $\mathrm{I}_{\mathrm{B}_{1}}$ & $\omega_{\mathrm{B}_{2}}$ & $\mathrm{I}_{\mathrm{B}_{2}}$ \\
\hline $\mathrm{VH}_{1}^{d}$ & $3188 \mathrm{~A}^{\prime}$ & 82 & - & - & $1487 \mathrm{~A}^{\prime}$ & 13 & $1421 \mathrm{~B}^{\prime \prime}$ & 32 \\
$\mathrm{VH}_{1}^{q}$ & $2797 \mathrm{~A} 1$ & 256 & - & - & $1356 \mathrm{E}$ & 106 & - & - \\
$\mathrm{VH}_{2}^{s}$ & $3605 \mathrm{~A}^{\prime}$ & 7 & $3404 \mathrm{~B}^{\prime \prime}$ & 13 & $1602 \mathrm{~A}^{i \prime}$ & 63 & $1399 \mathrm{~A}^{\prime}$ & 23 \\
$\mathrm{VH}_{2}^{t}$ & $3538 \mathrm{~A} 1$ & 1 & $3281 \mathrm{~B} 1$ & 31 & $1616 \mathrm{~A} 1$ & 21 & $1359 \mathrm{~B} 2$ & 62 \\
$\mathrm{VH}_{3}^{d}$ & $3909 \mathrm{~A} 1$ & 2 & $3647 \mathrm{E}$ & 0 & $1530 \mathrm{E}$ & 104 & $1485 \mathrm{~A} 1$ & 76 \\
$\mathrm{VH}_{4}$ & $4239 \mathrm{~A} 1$ & 0 & $3980 \mathrm{~F} 2$ & 4 & $1491 \mathrm{~F} 2$ & 224 & $1408 \mathrm{E}$ & 0 \\
\hline \hline
\end{tabular}

TABLE IV: Peak position $\left(\omega\right.$ in $\left.\mathrm{cm}^{-1}\right)$ and intensity $(I$ in $\mathrm{km} / \mathrm{mol}$ ) of the C-H stretching $(S)$ and bending ( $B$; only the ones above $1350 \mathrm{~cm}^{-1}$ are shown) modes. The symmetry of the mode and are also reported and in the bracket there's the intensities of the peaks obtained using a Lorentzian fitting with FWHM of $8 \mathrm{~cm}^{-1}$. 


\begin{tabular}{cccccccccccc}
\hline \hline $\mathrm{n} \mathrm{H}$ & $\mathrm{PG}$ & $\mathrm{IR}$ & $\mathrm{N}_{v}$ & $\omega_{e 1}$ & $\omega_{e 2}$ & $\omega_{e} \chi_{e}$ & $\omega_{e}$ & $\delta \omega_{e 1 e}$ & $\omega_{H}^{1}$ \\
\hline $\mathrm{VH}_{1}^{q}$ & $\mathrm{C}_{3 v}$ & $\mathrm{~A}_{1}$ & 1 & 2483 & 4631 & 168 & 2818 & -335 & 2797 \\
$\mathrm{VH}_{1}^{d}$ & $\sigma_{v}$ & $\mathrm{~A}^{\prime}$ & 1 & 3015 & 5814 & 108 & 3232 & -217 & 3188 \\
\hline $\mathrm{VH}_{2}^{t}$ & $\mathrm{C}_{2 v}$ & $\mathrm{~B}_{1}$ & 1 & 3253 & 6334 & 86 & 3426 & -173 & 3281 \\
$\mathrm{VH}_{2}^{t}$ & $\mathrm{C}_{2 v}$ & $\mathrm{~A}_{1}$ & 2 & 3477 & 6897 & 28 & 3533 & -56 & 3538 \\
$\mathrm{VH}_{2}^{s}$ & $\sigma_{v}$ & $\mathrm{~B}^{\prime \prime}$ & 1 & 3378 & 6617 & 70 & 3518 & -140 & 3404 \\
$\mathrm{VH}_{2}^{s}$ & $\sigma_{v}$ & $\mathrm{~A}^{\prime}$ & 2 & 3552 & 7050 & 27 & 3606 & -54 & 3605 \\
\hline $\mathrm{VH}_{3}^{d}$ & $\mathrm{C}_{3 v}$ & $\mathrm{E}$ & 1 & 3653 & 7202 & 53 & 3759 & -106 & 3647 \\
$\mathrm{VH}_{3}^{d}$ & $\mathrm{C}_{3 v}$ & $\mathrm{~A}_{1}$ & 3 & 3953 & 7921 & -8 & 3938 & 15 & 3909 \\
\hline $\mathrm{VH}_{4}^{s}$ & $\mathrm{~T}_{d}$ & $\mathrm{~F}_{2}$ & 1 & 4037 & 8021 & 27 & 4090 & -53 & 3980 \\
$\mathrm{VH}_{4}^{s}$ & $\mathrm{~T}_{d}$ & $\mathrm{~A}_{1}$ & 4 & 4434 & 8952 & -42 & 4349 & 85 & 4239 \\
\hline \hline
\end{tabular}

TABLE V: Harmonic and anharmonic C-H stretching (wavenumbers in $\mathrm{cm}^{-1}$ ) for the various $\mathrm{VH}_{n}^{i}$ defects, where s,d,t and q superscripts stand for singlet, doublet, triplet and quadruplet. $\omega_{e 1}$, $\omega_{e 2}, \omega_{e} \chi_{e}$ and $\omega_{e}$ are obtained from the numerical solution of the one-dimensional Schroedinger equation, and are the fundamental anaharmonic contribution, the first overtone, the anharmonic constant and the fundamental harmonic frequency. $\mathrm{N}_{v}$ is the number of hydrogen atoms that are involved in the anharmonic motion. When larger than 1, all atoms are moving in phase. PG is the point group of the system in the selected state, IR is the irreducible representation according to which the system is vibrating. $\omega_{H}^{1}$ and $\omega_{H}^{2}$ are the harmonic frequencies obtained by diagonalizing the full hessian matrix, that is built by using the one point or the two point formula shown in equation 2 and 3 respectively. $\delta \omega_{e 1 e}$ is the difference between $\omega_{e 1}$ and $\omega_{e}$, the harmonic and anarmhonic wavenumbers, both computed by solving the one-dimensional Schroedinger equation. 
1 Woods G. S and A. T. Collins. Infrared Absorption Spectra of Hydrogen Complexes in Type I Diamonds. J. Phys. Chem. Solids, 44(5):471-475, 1983.

2 T. Hainschwang, F. Notari, E. Fritsch, and L. Massi. Natural, Untreated Diamonds Showing the A, B and C Infrared Absorptions ("ABC Diamonds"), and the $\mathrm{H}_{2}$ Absorption. Diamond Relat. Mater., 15(10):1555-1564, 2006.

3 J. P. Goss, R. Jones, M. I. Heggie, C. P. Ewels, P. R. Briddon, and S. Öberg. Theory of Hydrogen in Diamond. Phys. Rev. B, 65(11):115207, 2002.

${ }^{4}$ E. Fritsch, T. Hainschwang, L. Massi, and B. Rondeau. Hydrogen-Related Optical Centers in Natural Diamond: an Update. New Diamond Frontier Carbon Technol., 17(2):63-89, 2007.

5 J. P. Goss, P. R. Briddon, V. Hill, R. Jones, and M. J. Rayson. Identification of the Structure of the $3107 \mathrm{~cm}^{-1}$ H-related Defect in Diamond. J. Phys. Condens. Matter, 26:145801, 2014.

6 J. J. Charette. Essai de Classification des Bandes d'Absorption Infrarouge du Diamant. Physica, 27(11):1061-1073, 1961.

7 C. E. Melton, A. A. Giardini, and C. A. Salotti. Observation of Nitrogen, Water, CarbonDioxide, Methane and Argon as Impurities in Natural Diamonds, 1972.

8 R. M. Chrenko, R. S. McDonald, and K. A. Darrow. Infrared spectra of diamond coat. Nature, 213:474-476, 1967.

9 J. P. Perdew and A. Zunger. Self-Interaction Correction to Density-Functional Approximations for Many-Electron Systems. Phys. Rev. B, 23(10):5048, 1981.

10 S. Salustro, F. Gentile, Ph. D'Arco, B. Civalleri, M. Reŕat, and R. Dovesi. Hydrogen Atoms in the Diamond Vacancy Defect. A Quantum Mechanical Vibrational Analysis. J. Chem. Phys., 15(10):1555-1564, 2006.

11 A. D. Becke. Density-Functional Thermochemistry. III. The Role of Exact Exchange. J. Chem. Phys., 98(7):5648-5652, 1993.

12 C. Lee, W. Yang, and R. G. Parr. Development of the Colle-Salvetti Correlation-Energy Formula into a Functional of the Electron Density. Phys. Rev. B, 37(2):785, 1988.

13 R. Dovesi, R. Orlando, A. Erba, C. M. Zicovich-Wilson, B. Civalleri, S. Casassa, L. Maschio, M. Ferrabone, M. De La Pierre, P. D’Arco, Y. Noël, M. Causà, M. Rérat, and B. Kirtman. CRYSTAL14: A Program for the Ab Initio Investigation of Crystalline Solids. Int. J. Quantum 
Chem., 114:1287, 2014.

14 W. J. Hehre, R. Ditchfield, and J. A. Pople. SelfConsistent Molecular Orbital Methods. XII. Further Extensions of GaussianType Basis Sets for Use in Molecular Orbital Studies of Organic Molecules. J. Chem. Phys., 56(5):2257-2261, 1972.

15 M. M. Francl, W. J. Petro, W. J. Hehre, J. S. Binkley, M. S. Gordon, D. J. DeFrees, and J. A. Pople. Self-Consistent Molecular Orbital Methods. XXIII. A Polarization-Type Basis Set for Second-Row Elements. J. Chem. Phys., 77(7):3654-3665, 1982.

16 P. Dirac. A Theory of Electrons and Protons. Proc. R. Soc. A, 126(801):360-365, 1930.

17 J. P. Perdew, K. Burke, and M. Ernzerhof. Generalized Gradient Approximation Made Simple. Phys. Rev. Lett., 77(18):3865-3868, 1996.

18 C. Adamo and V. Barone. Toward Chemical Accuracy in the Computation of NMR Shieldings: the PBE0 Model. Chem. Phys. Lett., 298(1):113-119, 1998.

19 A. V. Krukau, O. A. Vydrov, A. F. Izmaylov, and G. E. Scuseria. Influence of the Exchange Screening Parameter on the Performance of Screened Hybrid Functionals. J. Chem. Phys., 125(22):224106-224106, 2006.

20 R. Dovesi, V. R. Saunders, C. Roetti, R. Orlando, C. M. Zicovich-Wilson, F. Pascale, B. Civalleri, K. Doll, N. M. Harrison, I. J. Bush, Ph. D'Arco, and M. Llunell. CRYSTAL 2014 User's Manual. University of Torino, Torino, 2013.

21 F. Pascale, C. M. Zicovich-Wilson, F. Lòpez Gejo, B. Civalleri, R. Orlando, and R. Dovesi. The Calculation of the Vibrational Frequencies of the Crystalline Compounds and its Implementation in the CRYSTAL Code. J. Comput. Chem., 25(6):888-897, 2004.

22 C. M. Zicovich-Wilson, F. Pascale, C. Roetti, V. R. Saunders, R. Orlando, and R. Dovesi. Calculation of the Vibration Frequencies of $\alpha$-Quartz: The Effect of Hamiltonian and Basis Set. J. Comput. Chem., 25(15):1873-1881, 2004.

23 A. Erba, M. Ferrabone, R. Orlando, and R. Dovesi. Accurate Dynamical Structure Factors from Ab Initio Lattice Dynamics: the Case of Crystalline Silicon. J. Comput. Chem., 34:346-354, 2013.

24 C. Carteret, M. De La Pierre, M. Dossot, F. Pascale, A. Erba, and R. Dovesi. The Vibrational Spectrum of $\mathrm{CaCO}_{3}$ Aragonite: a Combined Experimental and Quantum-Mechanical Investigation. J. Chem. Phys., 138(1):014201, 2013.

25 J. Baima, M. Ferrabone, R. Orlando, A. Erba, and R. Dovesi. Thermodynamics and phonon 
dispersion of pyrope and grossular silicate garnets from ab initio simulations. Phys. Chem. Minerals, 43:137-149, 2016.

26 F. Pascale, C. M. Zicovich-Wilson, R. Orlando, C. Roetti, P. Ugliengo, and R. Dovesi. Vibration Frequencies of $\mathrm{Mg}_{3} \mathrm{Al}_{2} \mathrm{Si}_{3} \mathrm{O}_{12}$ Pyrope. An Ab Initio Study with the CRYSTAL Code. J. Phys. Chem. B, 109(13):6146-6152, 2005.

27 F. Pascale, P. Ugliengo, B. Civalleri, R. Orlando, Ph. DArco, and R. Dovesi. Hydrogarnet Defect in Chabazite and Sodalite Zeolites: A Periodic Hartree-Fock and B3-LYP Study. J. Chem. Phys., 117(11):5337-5346, 2002.

28 R. Orlando, F. J. Torres, F. Pascale, P. Ugliengo, C. M. Zicovich-Wilson, and R. Dovesi. Vibrational Spectrum of Katoite $\mathrm{Ca}_{3} \mathrm{Al}_{2}\left[(\mathrm{OH})_{4}\right]_{3}$ : a Periodic Ab Initio Study. J. Phys. Chem. $B, 110: 692-701,2006$.

29 F. Pascale, S. Tosoni, C. M. Zicovich-Wilson, P. Ugliengo, R. Orlando, and R. Dovesi. Vibrational Spectrum of Brucite $\mathrm{Mg}(\mathrm{OH})_{2}$ : a Periodic Ab Initio Quantum Mechanical Calculation Including OH Anharmonicity. Chem. Phys. Lett., 396:308-315, 2004.

30 S. Tosoni, F. Pascale, P. Ugliengo, R. Orlando, V. R. Saunders, and R. Dovesi. Quantum Mechanical Calculation of the OH Vibrational Frequency in Crystalline Solids. Molecular Physics, 103(18):2549-2558, 2005

31 R. Demichelis, Y. Nöel, B. Civalleri, C. Roetti, M. Ferrero, and R. Dovesi. The Vibrational Spectrum of $\alpha$-AlOOH Diaspore: an Ab Initio Study with the CRYSTAL Code. J. Phys. Chem. B, 111(31):9337-9346, 2007.

32 M. Mérawa, P. Labeguerie, P. Ugliengo, K. Doll, and R. Dovesi. The Structural, Electronic and Vibrational Properties of LiOH and NaOH: an Ab Initio Study. Chem. Phys. Lett., 387(4):453$459,2004$.

33 P. Ugliengo, F.Pascale, M. Merawa, P. Labéguerie, S. Tosoni, and R. Dovesi. Infrared Spectra of Hydrogen-Bonded Ionic Crystals: Ab Initio Study of $\mathrm{Mg}(\mathrm{OH})_{2}$ and $\beta-\mathrm{Be}(\mathrm{OH})_{2}$. J. Phys. Chem. B, 108(36):13632-13637, 2004.

34 G. M. Barrow. Introduction to molecular spectroscopy. McGraw-Hill, New York, 1962.

35 B. A. Hess, L. J. Schaad, P. Carsky, and R. Zahradnik. Ab Initio Calculations of Vibrational Spectra and Their Use in the Identification of Unusual Molecules. Chem. Rev, 86:709-730, 1986.

36 L. Maschio, B. Kirtman, R. Orlando, and M. Rérat. Ab initio Analytical Infrared Intensities for Periodic Systems Through a Coupled Perturbed Hartree-Fock/Kohn-Sham Method. J. Chem. 
Phys., 137(20):204113, 2012.

L. Maschio, B. Kirtman, M. Rérat, R. Orlando, and R. Dovesi. Comment on "Ab Initio Analytical Infrared Intensities for Periodic Systems Through a Coupled Perturbed Hartree-Fock/KohnSham Method" [J. Chem. Phys. 137, 204113 (2012)]. J. Chem. Phys., 139:167101, 2013. L. Maschio, B. Kirtman, M. Rérat, R. Orlando, and R. Dovesi. Ab Initio Analytical Raman Intensities for Periodic Systems Through a Coupled Perturbed Hartree-Fock/Kohn-Sham Method in an Atomic Orbital Basis. I. Theory. J. Chem. Phys., 139(16):164101, 2013.

39 L. Maschio, B. Kirtman, M. Rérat, R. Orlando, and R. Dovesi. Ab initio Analytical Raman Intensities for Periodic Systems Through a Coupled Perturbed Hartree-Fock/Kohn-Sham Method in an Atomic Orbital Basis. II. Validation and Comparison with Experiments. J. Chem. Phys., 139:164102, 2013.

40 M. Ferrero, M. Rérat, R. Orlando, and R. Dovesi. The Calculation of Static Polarizabilities of Periodic Compounds. The Implementation in the CRYSTAL code for 1D, 2D and 3D systems. J. Comput. Chem., 29:1450-1459, 2008.

41 M. Ferrero, M. Rérat, R. Orlando, and R. Dovesi. Coupled Perturbed Hartree-Fock for Periodic Systems: the Role of Symmetry and Related Computational Aspects. J. Chem. Phys., 128:014110, 2008.

42 D. A. McQuarrie and J. D. Simon. Physical Chemistry. A Molecular Approach. University Science Books, Sausalito, CA, 1977.

43 J. L. Duncan, D. C. McKean, I. Tortoand A. Brown, and A. M. Ferguson. Infrared Studies of $\mathrm{CH}$ and CD Stretching Anharmonicity. Journal of the Chemical Society, Faraday Transactions 2: Molecular and Chemical Physics, 84(9):1423-1442, 1988.

44 M. L. Myrick, A. E. Greer, A. A. Nieuwland, R. J. Priore, J. Scaffidi, D. Andreatta, and P. Colavita. Birge-Sponer Estimation of the $\mathrm{C}-\mathrm{H}$ Bond Dissociation Energy in Chloroform Using Infrared, Near-Infrared, and Visible Absorption Spectroscopy. An Experiment in Physical Chemistry. J. Chem. Educ, 85(9):1276, 2008.

45 P. Ugliengo. ANHARM. A Program to Solve Monodimensional Nuclear Schroedinger Equation. unpublished, 1989.

46 J. Baima, A. Zelferino, P. Olivero, A. Erba, and R. Dovesi. Raman Spectroscopic Features of the Neutral Vacancy in Diamond from Ab Initio Quantum-mechanical Calculations. Phys. Chem. Chem. Phys., 18(3):1961-1968, 2016. 
47 Alessandro Zelferino, Simone Salustro, Jacopo Baima, Valentina Lacivita, Roberto Orlando, and Roberto Dovesi. The electronic states of the neutral vacancy in diamond: a quantum mechanical approach. Theor. Chem. Acc., 135(3):1-11, 2016.

48 F. Gentile, S. Salustro, M. Causá, A. Erba, P. Carbonniére, and R. Dovesi. The $\mathrm{VN}_{3} \mathrm{H}$ Defect in Diamond. A Quantum Mechanical Investigation of the Structural, Electronic and Vibrational Properties. Phys. Chem. Chem. Phys., 111(44):11205-11213, 2017.

49 Benjamin Rondeau, Emmanuel Fritsch, Michel Guiraud, Jean-Pierre Chalain, and Franck Notari. Three Historical "Asteriated" Hydrogen-Rich Diamonds: Growth History and SectorDependent Impurity Incorporation. Diamond and Related Materials, 13(9):1658 - 1673, 2004. 